\title{
Compilation, Evaluation,
} and Prediction of Triple-Layer Model Constants for lons on Fe (III) and Mn (IV) Hydrous Oxides

R. W. Smith

E. A. Jenne

November 1988

Prepared for the

U.S. Environmental Protection Agency

under a Related Services Agreement

with the U.S. Department of Energy

under Contract DE-AC06-76RLO 1830

Pacific Northwest Laboratory

Operated for the U.S. Department of Energy

by Battelle Memorial Institute 
Although the research described in this article has been funded wholly or in part by the United States Environmental Protection Agency (EPA), it has not been subjected to EPA review and therefore does not necessarily reflect the views of EPA and no official endorsement should be inferred.

\title{
DISCLAIMER
}

This report was prepared as an account of work sponsorf $d$ by an agency of the United States Government. Neither the United States Government nor any agency thereof, nor Battelle Memorial Institute, nor any or their employees, makes any warranty, expressed or implied, or assumes any legal liability or responsibility for the accuracy, completeness, or usefulness of any information, apparatus, product, or process disclosed, or represents that its use would not infringe privately owned rights. Reference herein to any specific commercial product, process, or service by trade name, trademark, manufacturer, or otherwise does not necessarily constitute or imply its endorsement, recommendation, or favoring by the United States Government or any agency thereof, or Battelle Memorial Institute. The views and opinions of authors expressed herein do not necessarily state or reflect those of the United States Government or any agency thereof.

\author{
PACIFIC NORTHWEST LABORATCIRY \\ operated by \\ BATTELLE MEMORIAL INSTITUTE \\ for the \\ UNITED STATES DEPARTMENT OF ENERGY \\ under Contract DE-AC06-76RLO 1830
}

Printed in the United States of America
Available from
National Technical Information Service
United States Depar1ment of Commerce
5285 Port Royal Road
Springfield, Virginia 22161
NTIS Price Codes
Microfiche A01
Printed Copy
Pages
$001-025$
$026-050$
$051-075$


COMPILATION, EVALUATION, AND PREDICTION OF TRIPLE-LAYER MODEL CONSTANTS FOR IONS ON FE(III) AND Mn(IV) HYDROUS OXIDES

R.W. Smith

E.A. Jenne

November 1988

Prepared for the

U.S. Environmental Protection Agency under a Related Services Agreement with the U.S. Department of Energy Contract OE-ACO6-76RLO 1830

Pacific Northwest Laboratory

Richiand. Washington 99352 
= 


\section{SUMMARY}

The disposal of hazardous metals and metalloids is a significant source for the introduction of priority pollutants (Ag, As, Ba, Cd, Co, Cr. Cu, $\mathrm{Hg}$, $\mathrm{Pb}$. Sb. Se, $T l$, and $\mathrm{Zn}$ ) into groundwater systems. Adsorption onto iron and manganese oxides can retard the transport of these pollutants. The triplelayer model has been developed to quantify the adsorption of ions onto mineral surfaces. This report presents a literature review and an evaluation of triple-layer model acidity constants ( $\mathrm{pK}_{\mathrm{a} 1}^{\mathrm{int}}$, $p \mathrm{~K}_{\mathrm{a} 2}^{i n t}$; 21 vaiues) and intrinsic surface complexation constants ( $p^{\star} K^{\text {int }}$; 131 values) for ion adsorption onto $\alpha-\mathrm{FeOOH}$, amorphous $\mathrm{Fe}(\mathrm{III})$ oxide. and $\delta-\mathrm{MnO}_{2}$. The purpose of this evaluation of surface complexation constants was to provide a consistent set of triple-layer parameters to use in modeling the adsorption behavior of environmentally significant metals and metalloids in groundwater/sediment systems. In addition, constants for the major solute ions ( $\mathrm{Na}^{+}, \mathrm{K}^{+}, \mathrm{Ca}^{2+}$. $\mathrm{Mg}^{2+}, \mathrm{Cl}^{-}, \mathrm{CO}_{3}^{2}$, and $\mathrm{SO}_{4}^{2 \cdot}$ ) were tabulated to facilitate adsorption modeling of aquifers and vadose zones.

The range of the 19 acidity constants identified for amorphous fe(III) hydrous oxide and $\alpha-\mathrm{FeOOH}$ suggest that they are derived from a single norma] population with means of $5.0 \pm 0.5$ (1 standard deviation) and $10.9 \pm 0.5$ for $p_{a 1}^{i n t}$ and $p k_{a 2}^{i n t}$, respectively comitting the values of Balistrieri and Murray 1981). Furthermore, the most significant source of uncertainty in the reported acidity constants was the value for site density (the values vary by a factor of 6.5) used by different investigators. Two values of acidity constants were reported for $\delta$-Mn02. These two determinations were made on different materials, aged (BaTistrieri and Murray 1982b), and freshly precipitated (Catts and Langmuir 1986). Site density differed oy a factor of 12 between the two determinations.

Intrinsic constants for both cations and anions reported in the literature were recast in terms of surface complexation constants ( $\left.\log \mathrm{K}^{5 \mathrm{c}}\right)$ :

$$
\begin{aligned}
& \log K_{\text {cation }}^{\text {sc }}=p K_{a 2}^{i n t}-\log \beta_{1 n}-p^{\star K_{\text {cation }}^{i n t}} \\
& \log K_{\text {anion }}^{\text {sc }}=p^{\star K_{\text {anion }}^{i n t}}-p K_{a 1}^{i n t}+\log \beta_{1 n}
\end{aligned}
$$


where $\log \beta_{\text {In }}$ is the nth hydrolysis constant. This transformation reduced the variation between values determined by different investigators and facilitated the selection of recommended values. Variations in site loading with adsorbing metal and uncertainties in acidity constants were the major sources of variation in the values of $p^{\star} k^{i n t}$. Predictive equations based on ion size and hydrolysis behavior have been derived and values of $0^{\pi} k^{\text {int }}$ for important metals and metalloids predicted.

Evaluation of the correlations determined in this study suggest that significant improvements in both the accuracy of the experimental $0^{*} K^{i n t}$ values and our ability to predict triple-layer model constants would result from reevaluating the original experimental data. This should be done using consistent assumptions about site density and layer capacitance and common objective criteria to evaluate derivation of constants from experimental measurements. 


\section{ACKNOWLEDGMENTS}

This work was supported by the U.S. Environmental Protection Agency, Athens Environmental Research Laboratory under a Related Services Agreement with the U.S. Department of Energy under Contract DE-ACO6-76RLO 1830, Interagency Agreement DW90059-01. The authors would like to thank D. S. Brown and $N$. T. Loux for their interest and support, and $N$. T. Loux and J. P. Mcxinley for critical reviews and comment. Editorial assistance was provided by G. P. O.Connor. 


\section{CONTENTS}

SUMMARY

ACKNOWLEDGMENTS $\ldots \ldots \ldots \ldots \ldots \ldots \ldots \ldots \ldots \ldots \ldots$

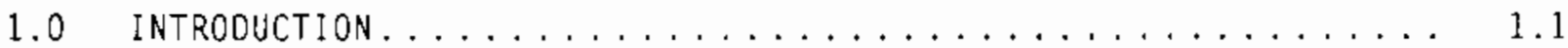

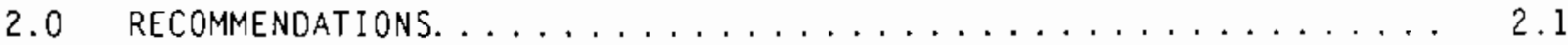

3.0 FORMULATION OF THE TRIPLE-LAYER MODEL $\ldots \ldots \ldots \ldots . \ldots \ldots$

4.0 SOURCES AND EVALUATION OF

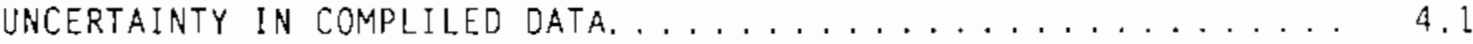

4.1 EFFECTS OF SITE CONCENTRATION

ON ACIDITY CONSTANTS AND $p^{\star} K^{i n t} \ldots \ldots \ldots \ldots . \ldots \ldots$

4.2 EFFECTS OF VARIABLE SITE OCCUPANCY ON $p^{\star} K^{i n t} \ldots \ldots \ldots . \ldots .10$

4.3 EFFECTS OF DATA REDUCTION METHOOS ON $p^{\star} k^{i n t} \ldots \ldots \ldots \ldots . . \ldots 42$

4.4 EFFECTS OF ATMOSPHERIC CARBON DIOXIDE ON $p^{\star K^{i n t}} \ldots \ldots \ldots 4.12$

4.5 EFFECTS OF INNER-SPHERE COMPLEX FORMATION ON $p^{\star} K^{i n t} \ldots \ldots 4.13$

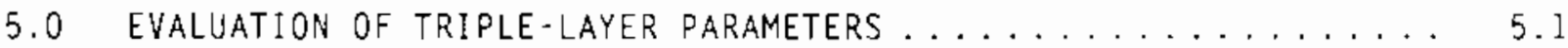

5.1 SURFACE ACIOITY CONSTANTS ............... 5.1

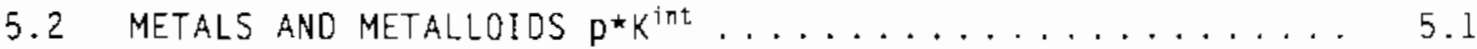

5.2 .1 Cation Adsorption on Fe(III) Hydrous 0xide...... 5.5

5.2 .2 Cation Adsorption on Mn(IV) 0xide ........ 5.7

5.2.3 Anion Adsorption on Fe(III) Hydrous Oxide....... 5.7

5.2 .4 Anion Adsorption on Mn(IV) oxide.......... 5.8

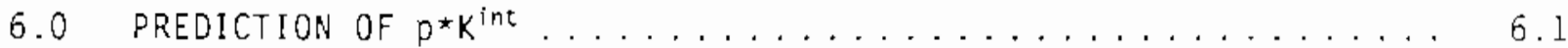

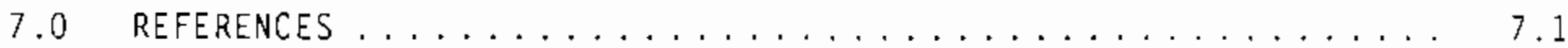

APPENDIX A - SUMMARY OF EXPERIMENTAL CONDITION............... 


\section{EIGURES}

3.1 Representation of the Oxide/Aqueous Electrolyte Interface Consistent with the Derivation of the Triple-Layer Model..... 3.2

4.1 Dependence of $\log K_{\mathrm{CCOH}}^{\mathrm{SC}}$ and $\log K_{\mathrm{HCrO4}}^{\mathrm{SC}}$. On the Experimental Adsorbate:Adsorbent Ratio.................4.11

6.1 Variation of $p^{\star} k^{i n t}$ with the Hydrolysis Constants for

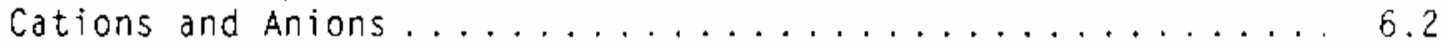

6.2 Representaion of Equation (6.4) and Equation (6.8) for the Prediction of Surface Complexation Constants ( $\log K^{\text {sc }}$ ) for Cations on Fe(III) Hydrous Oxides and Intinsic Surface Complexation Constants (log $K^{i n t}$ ) for Anion on $\mathrm{Fe}(1 \mathrm{II})$ Hydrous oxides................ 6.4

\section{TABLES}

4.1 Triple-Layer Model Parameters for Goethite. Amorphous Fe(III) Hydrous 0xide and Manganese 0xide ..............4.2

4.2 Triple-Layer Model Intrinsic Constants for Cation Adsorption on Amorphous Fe(III) Hydrous oxide in $0.1 \mathrm{M} \mathrm{NaNO}_{3} \ldots \ldots . . .4 .3$

4.3 Triple-Layer Model Intrinsic Constants for Cation Adsorption on Goethite in $0.1 \mathrm{M} \mathrm{NaNO}_{3} \ldots \ldots \ldots \ldots \ldots . \ldots \ldots$

4.4 Triple-Layer Model Intrinsic Constants for Cation Adsorption

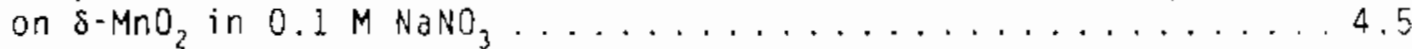

4.5 Triple-Layer Model Intrinsic Constants fo" Anion Adsorption on Amorphous Fe(III) Hydrous oxide in $0.1 \mathrm{M} \mathrm{NaNO}_{3} \ldots \ldots \ldots \ldots 4.6$

4.6 Definition of Reference Codes for Tables 4.1 through $4.5 \ldots \ldots 4$

5.1 Values of $g_{1}, g_{2}$, and Ionic Radius for Cations and Hydrolysis Constants................... 5.2

5.2 Surface Complexation Constants for Cations on Fe(III) Mydrous 0xide Substrates.................... 5.3

5.3 Surface Complexation Constants for Cations on Mn(IV) Oxide substrates......................... 5.4

5.4 Surface Complexation Constants for Anions on Fe(III) Hydrous 0xide Substrates......................... 5.4 


\section{TABLES (contd)}

6.1 Triple-Layer Model Intrinsic Complexation Constants for Fe(III) Hydrous oxides ....................... 6.7

6.2 Triple-Layer Model Intrinsic Complexation Constants

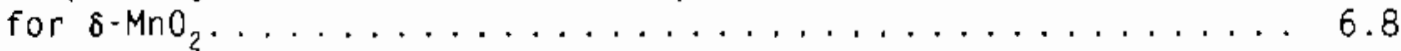


. 


\subsection{INTRODUCTION}

The land disposal of hazardous metals and metalloids is a significant source of priority pollutants (Ag, As, Bd, Cd, Co, Cr, Cu, Hg. Pb. Sb. Se, Tl and $2 n)$. To mitigate the potential hazard of these priority pollutants, the U.S. Environmental Protection Agency (EPA) is developing sediment quality criteria for metals and metalloids (Jenne et al. 1986; Crecelius, Jenne, and Anthony $1987^{(a)}$, and an adsorption database for priority pollutants. The sediment quality criteria will be used in conjunction with water quality criteria to protect aquatic organisms, the food chain to man, and drinking water from toxic metal contamination. The EPA is using geochemical models to calculate the speciation. solubility, and adsorption of metals and metalloids in the development of sediment quality criteria.

The triple-layer model (TLM) of the oxide/water interface is one computational method by which the partitioning of trace metals between sediments and aqueous phases can be numericaliy evaluated. This model requires that the activities of uncomplexed metal species be calculated. that the model-dependent constants (i.e.. the acidity constants and values of $p^{* k^{i n t}}$, for each pollutant be defined, and the surface properties of the sediment be characterized. The approach includes the thermodynamic calculation of metal speciation in groundwater and the calculation of the effects of solids by either adsorption/desorption mechanisms or dissolution/ precipitation mechanisms in limiting the release and transport of contaminants from a disposal site.

The EPA contracted with Pacific Northwest Laboratory (PNL) (b) to provide the site-independent information needed to evaluate the effect of adsorption/ desorption mechanisms. Specific tasks included compiling TLM parameters from the literature, evaluating the parameters, and selecting values for EPA to use in modeling pollutant transport in groundwater systems, and presenting

(a) Crecelius, E. A., E. A. Jenne, and J. S. Anthony. 1987. "Sediment Quality Criteria for Metals: Optimization of Extraction Methods for Determining the Quantity of Sorbents and Adsorbed Metals in Sediments." Submitted by Battelle. Washington Program affice, Washington. D.C. to the U.S. Environmental Protection Agency. Criteria and Standards Division.

(b) Operated by Battelle Memorial Institute for the U.S. Department of Energy. 
equations that can predict triple-layer parameters for selected metals and metalloids.

This report provides TLM parameters for $a-F e 00 H$, amorphous Fe(III) hydrous oxide, and $\delta-\mathrm{MnO}_{2}{ }$ (a) These solids were chosen because, along with organic matter, they are the major adsorbents in sediments and soils (Jenne 1977). Because these oxides have been extensively investigated using the TLM they are valuable in evaluating sources of uncertainty and developing predictive capability. Other solids such as $\mathrm{SiO}_{2}, \mathrm{TiO}_{2}$, and $\mathrm{Al}_{2} \mathrm{O}_{3}$ are not included in this report; however. Davis, James, and Leckie (1978) and Davis and Leckie (1978) provide several values of $p^{\star k^{i n t}}$ for them.

The EPA selected the TLM because of its applicability to varying pH and salinity conditions. However. several oxide/water interface models, in addition to the TLM, have been proposed by different investigators (see Barrow and Bowden 1987 for a summary). Comparisons among adsorption models are given by More1. Westa11, and Yeasted (1981): Sposito (1984): and Dzombak and More1 (1985) and are not repeated here. The conclusion of these investigators is that all the models can adequately reproduce a given set of experimental results and that the selection of which model to use is based on pragmatic considerations.

This report includes recommendations for further work in section 2.0. A brief discussion of the formulation of the TLM of the oxide/water interfaces is given in Section 3.0. Table of literature values for $p K_{a 1}^{i n t}, p K_{a 2}^{i n t}$, and $p^{\star} k^{i n t}$ are given in Section 4.0. In addition. the sources of uncertainties in TLM constants are discussed. Selected values of $p^{\star} K^{i n t}$ and $\log k^{s c}$ are given Section 5.0. Equations useful for the prediction of TLM parameters are presented in Section 6.0. Summaries of experimental conditions used by the various investigators referenced in this report are given in Appendix A.

(a) The results presented in this report compliment a similar study done by Dzombak (1986) for diffuse-layer model constants. 


\subsection{RECOMMENDATIONS}

This report indicates that the 1 iterature values of $p K_{a l}^{i n t}$ and $p K_{a 2}^{i n t}$ for Fe(III) hydrous oxides are normally distributed. which suggests that more accurate values of the acidity constant could be obtained if all experimental data were regressed, either step wise or simultaneously, using consistent assumptions about site densities and layer capacitances. Additionally, this effort has the potential to provide better definition to the site density concept as applied to amorphous material by allowing site density and surface area to be evaluated using capacitances and acidity constants determined from crystalline materials.

The methods used to derive values of $p^{\star} k^{i n t}$ are often poorly defined, subjective, and differ among investigators. Much of the uncertainty associated with $p^{\star} k^{i n t}$ could be removed if all the experimental work reported in the literature were re-evaluated using a single set of objective criteria for deriving values of $p^{\star} K^{i n t}$. In addition, the modifications to the $T L M$ of Hayes and Leckie (1987) could be incorporated. Two significant advantages exist for re-evaluating the work:

- a set of internaliy consistent constants with less uncertainty would be derived

- the ability to accurately predict constants for adsorbates would be greatiy improved.

The set of internally consistent constants and accompanying correlation plots would be used to assist in the design of an experimental program by:

- identifying literature results that are anomalous and require further experimental investigation

- prioritizing adsorbates for which no experimental work has been done. 
- 


\subsection{FORMULATION OF THE TRIPLE-LAYER MODEL}

The conceptual model of metal complexation reactions developed by Yates, Levine, and Healy (1974) and modified by Davis, James, and Leckie (1978) uses the TLM of the oxide/water interface. The TLM partitions the electrical double layer into two constant capacitance layers and an outer diffuse layer. The innermost plane, shown in Figure 3.1, contains only protons and hydroxyl ions and is characterized by a charge $\sigma_{0}$ and potential $\Psi_{0}$. Specific adsorption of ions occurs in the second $(\beta)$ plane with characteristic charge and potential $\sigma_{\beta}$ and $\Psi_{\beta}$, respectively. The specifically adsorbed ions are attracted to oppositely charged surface sites at the innermost plane. The third plane (with potential $\Psi_{d}$ and charge $\sigma_{d}$ ) marks the inner boundary of the diffuse layer. The capacitances $\left(C_{1}\right.$ and $\left.C_{2}\right)$ are given by:

$$
\begin{aligned}
& C_{1}=\frac{\sigma_{0}}{\Psi_{0}-\Psi_{\beta}} \\
& C_{2}=\frac{-\sigma_{d}}{\Psi_{\beta}-\Psi_{d}}
\end{aligned}
$$

The acid-base properties of an amphoteric oxide surface are described by two acid-base reactions:

$$
\begin{aligned}
& \mathrm{SOH}_{2}^{+} \stackrel{\mathrm{K}_{\mathrm{a} 1}^{\text {int }}}{=} \mathrm{SOH}^{0}+\mathrm{H}_{\mathrm{s}}^{+} \\
& \mathrm{SOH}^{0} \stackrel{\mathrm{K}_{\mathrm{a} 2}^{\text {int }}}{=} \mathrm{SO}^{-}+\mathrm{H}_{\mathrm{s}}^{+}
\end{aligned}
$$

where $S$ denotes a metal ion at the oxide surface: $\mathrm{SOH}_{2}^{+}$. $\mathrm{SOH}^{\circ}$, and $\mathrm{SO}^{-}$are the protonated, neutral, and deprotonated surface species, respectively: and surface plane protons are depicted by $\mathrm{H}_{\mathrm{s}}^{+}$. The concentration of protons at location $i$ in the electrical double layer is related to the bulk solution proton concentration by the Boltzmann distribution: 


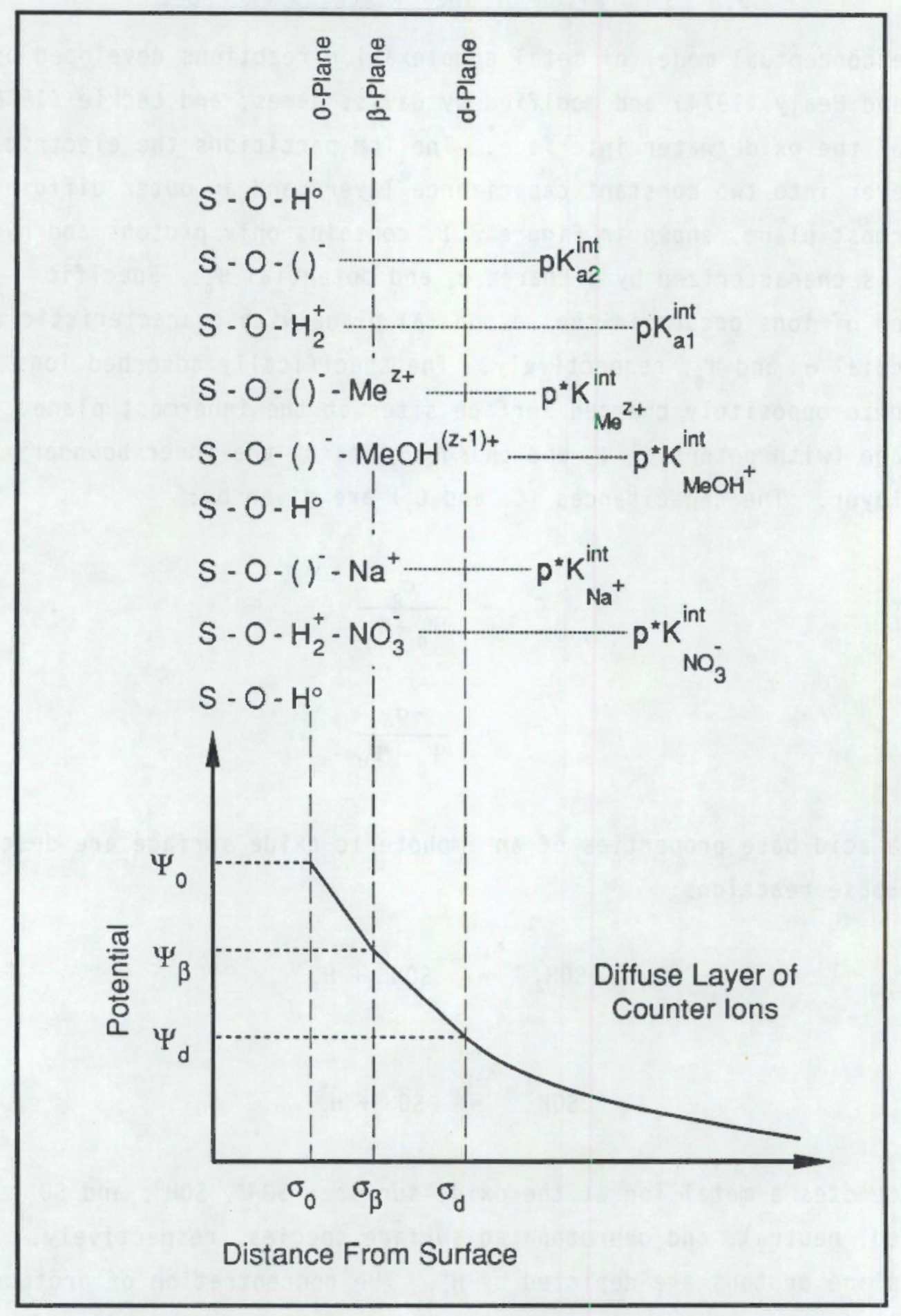

FIGURE 3.1. Representation of the Oxide/Aqueous Electrolyte Interface Consistent with the Derivation of the Triple-Layer Model 


$$
\left[H_{f}^{+}\right]=\left[H^{+}\right] \exp \left(\frac{-e \Psi_{0}}{k T}\right)
$$

Hence, for the $0-p l a n e$, the acidity constants becomes

$$
K_{\mathrm{a} 1}^{\text {int }}=\frac{\left[\mathrm{SOH}^{0}\right]\left[\mathrm{H}^{+}\right]}{\left[\mathrm{SOH}_{2}^{+}\right]} \exp \left(\frac{-e \Psi_{0}}{\mathrm{kT}}\right)
$$

and

$$
K_{\mathrm{a} 2}^{\mathrm{int}}=\frac{\left[S \mathrm{~S}^{\circ}\right]\left[\mathrm{H}^{+}\right]}{\left[\mathrm{SOH}^{0}\right]} \exp \left(\frac{-e \Psi_{0}}{\mathrm{kT}}\right)
$$

where $k$ is the Boltzmann constant, and $T$ is the temperature in kelvin. Similar pairs of expressions can be written for specific adsorption of cations and anions:

$$
\begin{aligned}
& \mathrm{SOH}^{\circ}+\mathrm{Na}^{+} \stackrel{\mathrm{Ka}^{+}}{=} \mathrm{SO}^{-}-\mathrm{Na}^{+}+\mathrm{H}^{+} \\
& \star \mathrm{K}_{\mathrm{Na}}^{i n t}=\frac{\left[\mathrm{SO}^{-}-\mathrm{Na}^{+}\right]\left[\mathrm{H}^{+}\right]}{\left[\mathrm{SOH}^{\circ}\right]\left[\mathrm{Na}^{+}\right]} \exp \left(\frac{e \Psi_{\beta^{*}}-e \Psi_{0}}{\mathrm{KT}}\right)
\end{aligned}
$$

and

$$
\begin{gathered}
\mathrm{SOH}_{2}^{+} \mathrm{Cl} \cdot \stackrel{{ }^{*} \mathrm{~K}_{\mathrm{Cl}}^{\mathrm{int}}}{=} \mathrm{SOH}+\mathrm{Cl}^{\circ}+\mathrm{H}^{+} \\
* \mathrm{~K}_{\mathrm{Cl}}^{\mathrm{int}}=\frac{\left[\mathrm{SOH}^{0}\right]\left[\mathrm{Cl}^{-}\right]\left[\mathrm{H}^{+}\right]}{\left[\mathrm{SOH}_{2}^{+}-\mathrm{Cl}^{-}\right]} \exp \left(\frac{e \Psi_{\beta}-e \Psi_{0}}{\mathrm{KT}}\right)
\end{gathered}
$$

where $\mathrm{SOH}_{2}^{+}-\mathrm{Cl}^{-}$represents a protonated surface site (2 protons in the 0 -plane) with a $\mathrm{Cl}^{-}$adsorbed in the $\beta-\mathrm{plane}$, and $\mathrm{SO}^{-}-\mathrm{Na}^{+}$represents a deprotonated surface site $\left(0\right.$ protons in the 0 -plane) with a $\mathrm{Na}^{+}$adsorbed in the $\beta$-plane. 
By convention, the reactions are written such that the hydrogen ion appears as a product (i.e.. acid dissociation). This convention leads to surface formation constants for cations and surface dissociation constants for anions. Detailed derivations of Equations (3.1) through (3.11) are given by Davis. James, and Leckie (1978) and Davis and Leckie (1978).

A recent modification to the TLM has been made to allow specific adsorption of ions to occur in both the 0 - and the $\beta$-planes (Hayes and Leckie 1987). This modification is based on the ionic strength dependency of adsorption and spectroscopic evidence (Hayes et i.l. 1987) for the formation of inner-sphere and outer-sphere complexes. The sicnificance of the modificatior is that it changes the predicted ionic strength dependency of adsorption for ions that form inner-sphere complexes, such as lead and cadmium. Results presented in this report are based on the originil model of Davis, james. Leckie (1978), which does not include provisions for the formation of inner-sphere and outer-sphere complexes. 


\subsection{SOURCES AND EVALUATION OF UNCERTAINTY IN COMPILED DATA}

Surface complexation models have been used to model the adsorption of metals onto sediments (Tessier, Rapin, and Carignan 1985) and blast furnace ash (Mihelcic and Luthy 1986), and phosphate onto soils (Goldberg and Sposito 1984). However, the usefulness of TLM $p^{\star} K^{i n t}$ values in setting criteria for the metal content of landfill leachates or the interstitial water of aquatic sediments is dependent on both the availability of the necessary database and the reliability of the individual TLM constants.

Surface parameters and acidity constants for Fe(III) hydrous oxides and a Mn(IV) oxide are summarized in Table 4.1. Reported values of $p^{\star} k^{i n t}$, acidity constants used in the calculation of these $p^{\star} x^{i n t}$ values, and experimental concentrations of adsorbate and adsorbent are presented in Tables 4.2 through 4.5. Literature sources for the constants presented in Tables 4.1 through 4.5 are given in Table 4.6. The experimental conditions used by various investigators are summarized in Appendix A. Major sources of the compiled data include: 1) Davis and Leckie (1978, 1980); Leckie et al. (1980). Benjamin and Bloom (1981) for amorphous Fe(III) hydrous oxide; 2) Balistrieri and Murray (1979; 1981; 1982a) for $\alpha-F e 00 H$; and 3) Catts and Langmuir (1986) and Balistrieri and Murray (1982b) and for $\delta-\mathrm{MnO}_{2}$. As Tables 4.2 through 4.5 demonstrate, large differences exist among the values of $p^{\star} k^{\text {int }}$ reported by these investigators. Identified sources of these differences include the:

- density and total number of adsorption sites on the oxide surface

- extent of occupancy (loading) of surface adsorption sites

- procedure for deriving $p^{\star} k^{i n t}$ from experimental adsorption data

- competition with $\mathrm{CO}_{3}^{2}$ and $\mathrm{HCO}_{3}$ resulting from dissolution of atmospheric $\mathrm{CO}_{2}$.

An additional source of uncertainty is the formation of inner-and outersphere complexes. The effects of these sources on the uncertainty of $p^{\star} K^{i n t}$ values is discussed in this section. 
IABLE 4.1. Triple-Layer Model Parameters for Goethite ( $\alpha-F e 00 H)$, Amorphous Fe(III) Hydrous Oxide and Manganese oxide $\left(\delta-\mathrm{MnO}_{2}\right)$

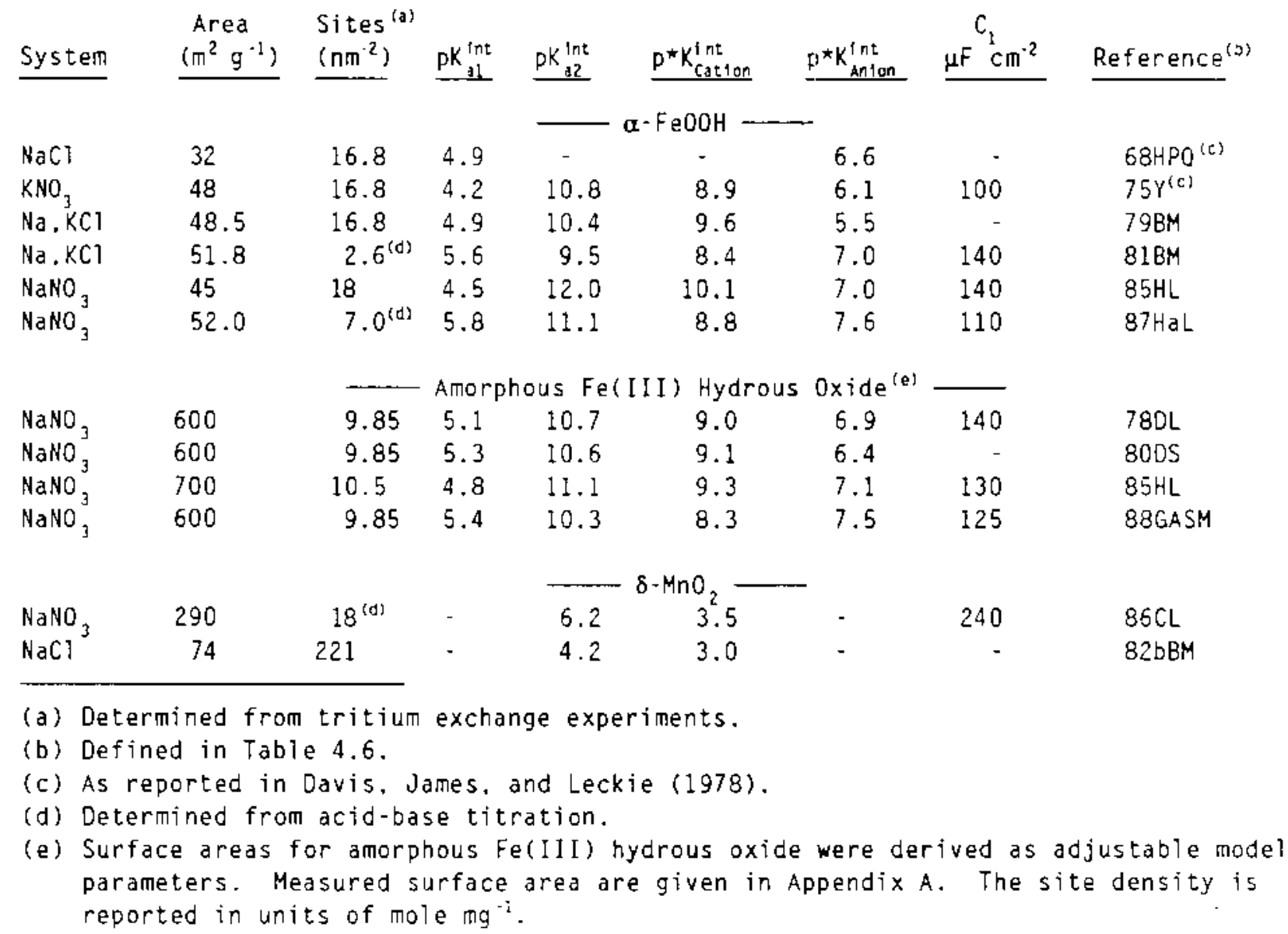


IABLE 4.2. Triple-Layer Model Intrinsic Constants for Cation Adsorption on Amorphous $\mathrm{Fe}(\mathrm{III})$ Hydrous oxide in $0.1 \mathrm{M} \mathrm{NaNO}_{3}$

\begin{tabular}{|c|c|c|c|c|c|c|c|c|}
\hline Species & $\begin{array}{l}\mathrm{Fe}_{\text {total }} \\
\log (\mathrm{mol})\end{array}$ & $\begin{array}{c}\text { Me } \text { total } \\
\left.1^{-1}\right)\end{array}$ & $p \star K_{\text {Cation }}^{t \pi t}$ & $\mathrm{pk}_{\mathrm{di}}^{\mathrm{int}}$ & $p K_{a 2}^{\ln t}$ & $p^{\star} K_{\mathrm{Nd}}^{1 n t}$ & $p \star K_{\mathrm{NO3}}^{i n t}$ & Reference (a) \\
\hline $\begin{array}{l}\mathrm{Ag}^{+} \\
\mathrm{Ag}(\mathrm{OH})^{\circ}\end{array}$ & -3.0 & -6.4 & $\begin{array}{r}5.0 \\
12.1\end{array}$ & 5.1 & 10.7 & 9.0 & 6.9 & $780 \mathrm{~L}$ \\
\hline $\mathrm{Ca}^{2+}$ & $\begin{array}{l}-(b) \\
-2.45\end{array}$ & -2.3 & $\begin{array}{l}6.3^{(0)} \\
10.5^{(0)}\end{array}$ & $\begin{array}{l}5.4 \\
5.3\end{array}$ & $\begin{array}{l}10.3 \\
10.6\end{array}$ & $\begin{array}{l}8.3 \\
9.1\end{array}$ & $\begin{array}{l}7.5 \\
6.4\end{array}$ & $\begin{array}{l}87 Z G S R \\
800 S\end{array}$ \\
\hline $\begin{array}{l}\mathrm{Cd}^{2+} \\
\mathrm{Cd}(\mathrm{OH})^{+}\end{array}$ & $\begin{array}{l}-2.0 \\
-3.0\end{array}$ & $\begin{array}{l}-5.0 \\
-6.3 \\
-5.0 \\
-6.3 \\
-7.7 \\
-6.3 \\
-5.7 \\
-4.3\end{array}$ & $\begin{array}{c}4.8 \\
4.9 \\
11.25 \\
11.0 \\
10.4 \\
10.6 \\
10.9 \\
11.2\end{array}$ & 5.1 & 10.7 & 9.0 & 6.9 & $\begin{array}{l}81 \mathrm{BB}^{(e)} \\
78 \mathrm{DL}^{(e)} \\
81 \mathrm{BB} \\
78 \mathrm{DL}^{(e)} \\
7 \mathrm{BB}\end{array}$ \\
\hline $\begin{array}{l}\mathrm{Co}^{2+} \\
\mathrm{Co}(\mathrm{OH})^{+}\end{array}$ & -3.0 & -5.0 & $\begin{array}{r}4.8 \\
11.6\end{array}$ & 5.1 & 10.7 & 9.0 & 6.9 & $81 \mathrm{BB}$ \\
\hline $\begin{array}{l}\mathrm{Cu}^{2+} \\
\mathrm{Cu}(\mathrm{OH})^{+}\end{array}$ & -3.0 & $\begin{array}{l}-6.0 \\
-6.3\end{array}$ & $\begin{array}{l}4.1 \\
9.0 \\
8.7\end{array}$ & 5.1 & 10.7 & 9.0 & 6.9 & $\begin{array}{l}780 \mathrm{~L} \\
78 \mathrm{~B}\end{array}$ \\
\hline $\mathrm{NpO}_{2}^{+}$ & $-(f)$ & $-(g)$ & $3.5^{(n)}$ & 5.4 & 10.3 & 8.3 & 7.5 & B8GASM \\
\hline $\begin{array}{l}\mathrm{Pb}^{2+} \\
\mathrm{Pb}(\mathrm{OH})^{+}\end{array}$ & -3.0 & -6.3 & $\begin{array}{l}3.8 \\
7.3 \\
7.3\end{array}$ & 5.1 & 10.7 & 9.0 & 6.9 & $\begin{array}{l}780 \mathrm{~L} \\
78 \mathrm{~B}\end{array}$ \\
\hline $2 n^{2+}$ & $\begin{array}{r}-3.0 \\
-3.8\end{array}$ & $\begin{array}{r}-5.0 \\
-(1)\end{array}$ & $\begin{array}{l}4.8 \\
2.3\end{array}$ & $\begin{array}{l}5.1 \\
5.3\end{array}$ & $\begin{array}{l}10.7 \\
10.6\end{array}$ & $\begin{array}{l}9.0 \\
9.1\end{array}$ & $\begin{array}{l}6.9 \\
6.4\end{array}$ & $\begin{array}{l}81 B B \\
800 S\end{array}$ \\
\hline $\mathrm{Zn}(\mathrm{OH})^{*}$ & -3.0 & $\begin{array}{l}-5.0 \\
-6.3\end{array}$ & $\begin{array}{l}10.5 \\
10.5 \\
10.3 \\
10.3\end{array}$ & 5.1 & 10.7 & 9.0 & 6.9 & $\begin{array}{l}818 \mathrm{~B} \\
78 \mathrm{~B} \\
780 \mathrm{~L}(\mathrm{e})\end{array}$ \\
\hline
\end{tabular}

(a) Defined in Table 4.6.

(b) -1.76 to -3.06 .

(c) -2.60 to -4.00 .

(d) $2\left(\mathrm{SOH}^{\circ}\right)+\mathrm{Ca}^{2+}=\left(\mathrm{SO}^{-}\right), \mathrm{Ca}^{2+}+2 \mathrm{H}^{*}$.

(e) Calculated from $\log K^{5 c}$ given by Davis and Leckie (1978).

(f) -2.0 to -3.0 .

(g) -10.35 to -12.35 .

(h) $\mathrm{SOH}^{\circ}+\mathrm{NpO}_{?}^{+}+\mathrm{H}_{2} \mathrm{O}=\mathrm{SOH}^{\circ}-\mathrm{NpO}_{2} \mathrm{OH}^{\circ}+\mathrm{H}^{+}$.

(i) -5.04 to -6.70 . 
IABLE 4.3. Triple-Layer Model Intrinsic Constants for Cation Adsorption on Goethite $(\boldsymbol{a}-\mathrm{FeOOH})$ in $0.1 \mathrm{M} \mathrm{NaNO}_{3}$ (except as noted)

\begin{tabular}{|c|c|c|c|c|c|c|c|c|}
\hline Species & $\begin{array}{l}F e_{\text {total }} \\
\log \text { (mol }\end{array}$ & $\begin{array}{c}M e_{\text {total }} \\
\left.l^{-1}\right) \\
\end{array}$ & $P^{\star} K_{\text {Cat1an }}^{1 \text { nt }}$ & $\mathrm{pK}_{\mathrm{al}}^{1 \mathrm{nt}}$ & $\mathrm{pK}_{\mathrm{d} 2}^{\operatorname{lnt}}$ & $\underline{P}^{*} K_{* a}^{i n t}$ & $P^{\star K_{\mathrm{NO3}}^{1 \mathrm{nt}}}$ & Reference $^{\text {(a) }}$ \\
\hline \multirow{2}{*}{$\begin{array}{l}\mathrm{Ca}^{2+} \\
\mathrm{CaOH}^{+}\end{array}$} & -1.07 & $\begin{array}{l}-(b) \\
-(c)\end{array}$ & $\begin{array}{c}5.0 \\
15.8^{(d)}\end{array}$ & $\begin{array}{l}5.57 \\
4.9\end{array}$ & $\begin{array}{l}9.52 \\
10.4\end{array}$ & 8.40 & $\begin{array}{l}7.00 \\
5.5\end{array}$ & $\begin{array}{l}81 B M \\
79 B M\end{array}$ \\
\hline & -1.07 & $-^{(b)}$ & $\begin{array}{l}16.5 \\
14.5\end{array}$ & 5.57 & 9.52 & 8.40 & 7.00 & $81 B M$ \\
\hline $\begin{array}{l}\mathrm{Cd}^{2+} \\
\mathrm{Cd}(\mathrm{OH})^{+}\end{array}$ & - & -5.55 & $\begin{array}{l}1.3^{(\mathrm{e})} \\
9.35\end{array}$ & 5.57 & 9.52 & 8.40 & 7.00 & $82 \mathrm{aBM}$ \\
\hline $\begin{array}{l}\mathrm{Cu}^{2+} \\
\mathrm{Cu}(\mathrm{OH})^{+}\end{array}$ & - & .5 .60 & $\begin{array}{l}3.0^{(\mathrm{e})} \\
7.0\end{array}$ & 5.57 & 9.52 & 8.40 & 7.00 & $82 \mathrm{aBM}$ \\
\hline \multirow{2}{*}{$\begin{array}{l}\mathrm{Mg}^{2+} \\
\mathrm{MgOH}^{+}\end{array}$} & -1.07 & $\begin{array}{l}-(f) \\
-(q)\end{array}$ & $\begin{array}{l}5.45 \\
11.9^{\text {(d) }}\end{array}$ & $\begin{array}{l}5.57 \\
4.9\end{array}$ & $\begin{array}{l}9.52 \\
10.4\end{array}$ & 8.40 & $\begin{array}{l}7.00 \\
5.5\end{array}$ & $\begin{array}{l}81 B M \\
79 B M\end{array}$ \\
\hline & -1.07 & $-(f)$ & $\begin{array}{l}12.2 \\
14.25\end{array}$ & 5.57 & 9.52 & 8.40 & 7.00 & $81 B M$ \\
\hline $\begin{array}{l}\mathrm{Pb}^{2+} \\
\mathrm{Pb}(\mathrm{OH})^{+}\end{array}$ & - & -5.48 & $\begin{array}{l}1.8^{(e)} \\
5.0\end{array}$ & 5.57 & 9.52 & 8.40 & 7.00 & $82 \mathrm{aBM}$ \\
\hline $\begin{array}{l}\mathrm{PuOH} \mathrm{H}^{3+} \\
\mathrm{Pu}(\mathrm{OH})_{2}^{2+}\end{array}$ & -2.21 & -11.0 & $\begin{array}{r}-2.5 \\
2.0\end{array}$ & 5.57 & 9.52 & 8.40 & . & B5SMS \\
\hline $\mathrm{Pu}(\mathrm{OH})_{3}^{+}$ & & & 5.9 & & & & & \\
\hline $\mathrm{Pu}(\mathrm{OH})_{4}^{\frac{3}{3}}$ & & & 12.0 & & . & & ${ }^{\circ}$ & \\
\hline $\begin{array}{l}\mathrm{UO}_{2} \mathrm{OH}^{+} \\
\left(\mathrm{UO}_{2}\right)_{3}(\mathrm{OH})_{5}^{+}\end{array}$ & -1.95 & -5.0 & $\begin{array}{c}8.0 \\
15.0^{(h)}\end{array}$ & 4.5 & 12.0 & 10.1 & 7.0 & $85 \mathrm{HL}$ \\
\hline $\mathrm{NO}_{2}\left(\mathrm{CO}_{3}\right)_{2}^{2-}$ & & $-5.0^{(f)}$ & $-30.0^{(j)}$ & & & & & \\
\hline \multirow[t]{2}{*}{$\mathrm{UO}_{2}\left(\mathrm{CO}_{3}\right)_{3}^{4-}$} & & $\begin{array}{l}-5.0^{(k)} \\
-5.0^{(1)}\end{array}$ & $\begin{array}{l}-29.5^{(j)} \\
+38.5^{(j)}\end{array}$ & & & & & \\
\hline & & $-5.0^{(k)}$ & $-42.5^{(j)}$ & & & & & \\
\hline $\mathrm{Zn}(\mathrm{OH})^{+}$ & . & -5.57 & (e) 9.15 & 5.57 & 9.52 & 8.40 & 7.00 & $82 \mathrm{aBM}$ \\
\hline
\end{tabular}

\footnotetext{
(a) Defined in Table 4.6.

(b) $\mathrm{CaCl} 2_{2}-\mathrm{NaCl}$ with $\mathrm{CaCl}_{2}$ : NaCl ratios of $10^{-4.63}: 10^{\cdot 3}, 10^{-3.42}: 10^{-3}$, and $10^{-3.4}: 10^{-2}$.

(c) 0.01 to $0.5 \mathrm{M} \mathrm{CaCl}_{2}$.

(d) $\left.2(\mathrm{~S}-\mathrm{OH})+\mathrm{M}^{2+}=\left[\left(\mathrm{S}-\mathrm{O}^{-}\right)\right)_{2}-\mathrm{H}^{2+}\right]+2 \mathrm{H}^{+}$.

(e) Determined in $0.53 \mathrm{M} \mathrm{NaCl}$.

(f) $\mathrm{MgCl}_{2}{ }^{-\mathrm{NaCl}}$ with $\mathrm{MgCl}_{2}$ : $\mathrm{NaCl}$ ratios of $10^{-4.24}: 10^{-3}, 10^{-4.23}: 10^{-2}, 10^{-4}: 0.53$, and $10^{-3.55}: 0.3$.

(g) 0.001 to $0.5 \mathrm{M} \mathrm{MgCl}_{2}$.

(h) Hsi and Langmuir (1985) also give values for bidentate and tridentate surface complexes that fit the experfmental results as well as the constants given here.

(i) $10^{2}$ total carbonate.

(j) $\left[\mathrm{SOH}_{2}^{+}-\mathrm{NO}_{2}\left(\mathrm{CO}_{3}\right)^{2-2 \mathrm{n}}\right]=\mathrm{SOH}^{\circ}+1 \mathrm{OO}_{2}^{2+}+\mathrm{nCO}_{3}^{2 \cdot}+\mathrm{H}^{+}$.

(k) $10^{-3}$ total carbonate.
} 
IABLE 4.4. Triple-Layer Model Intrinsic Constants for Cation Adsorption on $\delta-\mathrm{MnO}_{2}$ in $0.1 \mathrm{M} \mathrm{NaNO}_{3}$ (except as noted)

\begin{tabular}{|c|c|c|c|c|c|c|c|c|}
\hline Species & $\begin{array}{l}\text { Mn }{ }_{\text {total }} \\
\log (\mathrm{mol}\end{array}$ & $\begin{array}{c}\mathrm{Me}_{\text {(otal }} \\
\left.l^{-1}\right\rangle \\
\end{array}$ & $p^{\star} K_{\text {Cation }}^{1 \text { nt }}$ & $\mathrm{pK}_{\mathrm{a} 1}^{\ln t}$ & $\mathrm{pK}_{\mathrm{a} 2}^{\mathrm{int}}$ & $p^{\star} K_{\mathrm{Na}}^{1 \pi t}$ & $\mathrm{P}^{\star K_{\mathrm{NO3}}^{4 \mathrm{nt}}}$ & Reference \\
\hline $\mathrm{Ca}^{2+}$ & . & $-(b)$ & $3.3^{(c)}$ & - & 4.2 & 3.0 & - & $82 b B M$ \\
\hline $\begin{array}{l}\mathrm{Cu}^{2+} \\
\mathrm{Cu}(\mathrm{OH})^{+} \\
\mathrm{Cu}(\mathrm{OH})_{2}^{\circ}\end{array}$ & -4.14 & -5.0 & $\begin{array}{r}0.1 \\
7.5 \\
13.4\end{array}$ & - & 6.2 & 3.5 & - & $86 \mathrm{CL}$ \\
\hline $\mathrm{CuNO}_{3}^{+}$ & & & -1.8 & & & & & \\
\hline$K^{+}$ & - & $-(d)$ & 2.0 & - & 4.2 & 3.0 & . & $82 \mathrm{bBM}$ \\
\hline $\mathrm{Mg}^{2+}$ & - & (e) & $3.9^{(c)}$ & - & 4.2 & 3.0 & - & $82 \mathrm{bBM}$ \\
\hline $\begin{array}{l}\mathrm{Pb}^{2+} \\
\mathrm{Pb}(\mathrm{OH})^{+}\end{array}$ & -4.14 & -5.0 & $\begin{array}{r}-1.8 \\
6.5\end{array}$ & - & 6.2 & 3.5 & - & $86 \mathrm{CL}$ \\
\hline $\begin{array}{l}\mathrm{Zn}{ }^{2+} \\
\mathrm{Zn}(\mathrm{OH})^{+} \\
\operatorname{Zn}(\mathrm{OH})_{2}^{0}\end{array}$ & -4.14 & -5.0 & $\begin{array}{r}1.5 \\
8.8 \\
15.0\end{array}$ & - & 6.2 & 3.5 & - & $86 \mathrm{CL}$ \\
\hline $\mathrm{ZnNO}_{3}^{+}$ & & & -1.0 & & & & & \\
\hline
\end{tabular}
(a) Defined in Table 4.6 .
(b) $10^{-2}$ to $10^{-4} \mathrm{M} \mathrm{CaCl}_{2}$ and major ion seawater.
(c) $2\left(\mathrm{SOH}^{\circ}\right)+\mathrm{M}^{2+}=\left[\left(\mathrm{SO}^{-}\right) \mathrm{2}^{-\mathrm{M}^{2+}}\right]+2 \mathrm{H}^{+}$.
(d) $10^{-2}$ to $10^{-3} \mathrm{M} \mathrm{KCl}$.
(e) $10^{-2}$ to $10^{-4} \mathrm{M} \mathrm{MgCl}_{2}$ and major ion seawater. 
IABLE 4.5. Triple-Layer Model Intrinsic Constants for Anion Adsorption on Amorphous $\mathrm{Fe}(\mathrm{III})$ Hydrous oxide in $0.1 \mathrm{M} \mathrm{NaNO}_{3}$

\begin{tabular}{|c|c|c|c|c|c|c|c|c|}
\hline Species & $\begin{array}{l}F e_{\text {total }} \\
\log \text { (mo) }\end{array}$ & $\begin{array}{l}\text { Me }{ }_{\text {total }} \\
\left.\gamma^{-1}\right) \\
\end{array}$ & $P^{\star K_{\text {anlan }}^{1 \mathrm{nt}}}$ & $p K_{a 1}^{\text {int }}$ & $\mathrm{pK}_{\mathrm{a} 2}^{\mathrm{int}}$ & $P^{\star} K_{\mathrm{Nat}}^{1 \pi \mathrm{t}}$ & $P^{\star} K_{\mathrm{NO3}}^{i n t}$ & Reference \\
\hline \multirow[t]{2}{*}{$\mathrm{HASO}_{4}^{2}$} & -3.0 & -6.3 & 25.9 & 5.1 & 10.7 & 9.0 & 6.9 & 80LBHKA \\
\hline & $\begin{array}{l}-3.3 \\
-3.0\end{array}$ & $\begin{array}{r}-4.3 \\
-4.3 \\
-(b)\end{array}$ & $\begin{array}{l}25.0 \\
27.7 \\
31.1\end{array}$ & & & & & $\begin{array}{l}81 \mathrm{BB} \\
80 \mathrm{LBHKA}\end{array}$ \\
\hline $\mathrm{H}_{2} \mathrm{ASO}_{4}$ & -3.3 & -4.3 & 33.5 & & & & & $81 \mathrm{BB}$ \\
\hline \multirow{2}{*}{$\begin{array}{l}\mathrm{HCO}_{3}^{-} \\
\mathrm{H}_{2} \mathrm{CO}_{3}^{\mathrm{O}}\end{array}$} &.$(c)$ & -5.34 & 20.7 & 5.4 & 10.3 & 8.6 & 7.5 & 87 ZGSR \\
\hline & & & 20.0 & & & & & \\
\hline \multirow[t]{2}{*}{$\mathrm{CrO}_{\Delta}^{2-}$} & $-(c)$ & -5.3 & 10.1 & 5.4 & 10.3 & 8.6 & 7.5 & $87 Z G S R$ \\
\hline & -3.0 & $\begin{array}{l}-4.3 \\
-5.0 \\
-4.0\end{array}$ & $\begin{array}{l}10.6 \\
10.6 \\
11.9 \\
14.4\end{array}$ & 5.1 & 10.7 & 9.0 & 6.9 & $\begin{array}{l}80 L B H K A \\
800 L \\
81 B B\end{array}$ \\
\hline \multirow[t]{3}{*}{$\mathrm{HCrO}_{4}$} & -1.6 & -6.3 & $\begin{array}{r}9.8^{(d)} \\
19.4^{(d)}\end{array}$ & 4.2 & 10.8 & 8.9 & 6.1 & B8AGZS \\
\hline & -3.0 & $\begin{array}{l}-4.3 \\
-5.0\end{array}$ & $\begin{array}{l}18.1 \\
18.1 \\
18.0\end{array}$ & 5.1 & 10.7 & 9.0 & 6.9 & $\begin{array}{l}\text { 80LBHKA } \\
800 \mathrm{~L} \\
818 \mathrm{~B}\end{array}$ \\
\hline & $-(c)$ & $\begin{array}{l}-4.0 \\
-5.3\end{array}$ & $\begin{array}{l}16.8 \\
19.3\end{array}$ & 5.4 & 10.3 & 8.6 & 7.5 & $87 Z G S R$ \\
\hline $\mathrm{S}_{2} \mathrm{O}_{3}^{2-}$ & -3.0 & -4.3 & 10.0 & 5.1 & 10.7 & 9.0 & 6.9 & $80 \mathrm{LBHKA}$ \\
\hline & & & & & & & & \\
\hline \multirow[t]{2}{*}{$\mathrm{SO}_{4}^{2-}$} & $-(c)$ & $-2.6^{(e)}$ & 11.6 & 5.4 & 10.3 & 8.6 & 7.5 & $87 Z G S R$ \\
\hline & -3.0 & $\begin{array}{l}-5.0 \\
-4.3\end{array}$ & $\begin{array}{l}9.9 \\
9.9\end{array}$ & 5.1 & 10.7 & 9.0 & 6.9 & $\begin{array}{l}\text { 80DL } \\
\text { 80LBHKA }\end{array}$ \\
\hline \multirow[t]{2}{*}{$\mathrm{HSO}_{4}^{-}$} & $\begin{array}{l}-1.07 \\
-3.0\end{array}$ & $\begin{array}{l}-(f) \\
-4.3\end{array}$ & $\begin{array}{c}9.1^{(d)} \\
15.9\end{array}$ & $\begin{array}{l}5.6 \\
5.1\end{array}$ & $\begin{array}{r}9.5 \\
10.7\end{array}$ & $\begin{array}{l}8.4 \\
9.0\end{array}$ & $\begin{array}{l}7.0 \\
6.9\end{array}$ & $\begin{array}{l}\text { 81BM } \\
\text { 80LBHKA }\end{array}$ \\
\hline & $\begin{array}{l}-(c) \\
-1.07\end{array}$ & $\begin{array}{l}-5.0 \\
-2.6^{(e)} \\
-i f) \\
-(g)\end{array}$ & $\begin{array}{l}15.9^{17} \\
17.3^{(d)} \\
14.4^{(d)} \\
14.4^{(d)}\end{array}$ & $\begin{array}{l}5.4 \\
5.6 \\
4.9\end{array}$ & $\begin{array}{r}10.3 \\
9.5 \\
10.4\end{array}$ & $\begin{array}{l}8.6 \\
8.4 \\
9.6\end{array}$ & $\begin{array}{l}7.5 \\
7.0 \\
-\end{array}$ & $\begin{array}{l}80 D L \\
87 Z G S R \\
81 \mathrm{BM} \\
79 \mathrm{BM}\end{array}$ \\
\hline \multirow[t]{2}{*}{$\mathrm{SeO}_{4}^{2-}$} & -3.0 & -4.3 & 9.9 & 5.1 & 10.7 & 90 & 6.9 & 80LBHKA \\
\hline & & $\begin{array}{l}-5.0 \\
-4.0 \\
-3.0\end{array}$ & $\begin{array}{r}9.9 \\
11.8 \\
15.0\end{array}$ & & & & & $\begin{array}{l}800 \mathrm{~L} \\
81 \mathrm{BB}\end{array}$ \\
\hline \multirow[t]{2}{*}{$\mathrm{HSeO}_{4}^{-}$} & & -4.3 & 15.9 & & & & & 80LBHKA \\
\hline & & $\begin{array}{l}-5.0 \\
-4.0 \\
-3.0\end{array}$ & $\begin{array}{l}15.9 \\
15.6 \\
19.0\end{array}$ & & & & & $\begin{array}{l}800 \mathrm{~L} \\
818 \mathrm{~B}\end{array}$ \\
\hline \multirow[t]{2}{*}{$\mathrm{SeO}_{3}^{2-}$} & -3.0 & -6.3 & 12.5 & 5.1 & 10.7 & 9.0 & 6.9 & 8OLBHKA \\
\hline & $\begin{array}{l}-3.4 \\
-3.0\end{array}$ & $\begin{array}{l}-4.3 \\
-4.0 \\
-3.0\end{array}$ & $\begin{array}{l}11.4 \\
12.8 \\
12.9\end{array}$ & & & & & $81 \mathrm{BB}$ \\
\hline
\end{tabular}


TABLE 4.5. (contd)

\begin{tabular}{|c|c|c|c|c|c|c|c|c|}
\hline Species & $\begin{array}{l}\mathrm{Fe}_{\text {total }} \\
\log (\mathrm{mol})\end{array}$ & $\begin{array}{l}\mathrm{Me}_{\text {tatal }} \\
\left.\mathrm{l}^{-1}\right) \\
\end{array}$ & $p^{\star} K_{\text {Anion }}^{i n t}$ & $\mathrm{pK}_{\mathrm{d1}}^{\mathrm{int}}$ & $\mathrm{pK}_{\mathrm{a} 2}^{\ln \mathrm{t}}$ & $\mathrm{P}^{\star K_{\mathrm{Na}}^{i n t}}$ & $p^{\star} K_{\mathrm{NO3}}^{i n t}$ & Reference \\
\hline \multirow[t]{2}{*}{$\mathrm{HSeO}_{3}$} & -3.4 & -4.3 & 18.3 & & & & & 80LBHKA \\
\hline & -3.0 & $\begin{array}{l}-6.3 \\
-4.0 \\
-3.0\end{array}$ & $\begin{array}{l}18.9 \\
20.8 \\
22.0\end{array}$ & & & & & $81 \mathrm{BB}$ \\
\hline
\end{tabular}

\footnotetext{
(a) Defined in Table 4.6 .

(b) -4.3 to -6.3 .

(c) -1.76 to -3.06 .

(d) $\alpha-\mathrm{FeOOH}$.

(e) In addition $10^{-5.3} \mathrm{M} \mathrm{CrO}_{4}{ }^{2-}$ and $\mathrm{P}_{\mathrm{toz}}=10^{-2.46}$ was include in the experiment.

(f) $\mathrm{Na}_{2} \mathrm{SO}_{4}-\mathrm{NaCl}$ with $\mathrm{Na}_{2} \mathrm{SO}_{4}: \mathrm{NaCl}$ ratios of $10^{-3.43}: 10^{-2}$ and $10^{-3.68}: 0.53$.

(g) 0.001 to $0.53 \mathrm{M} \mathrm{Na}_{2} \mathrm{SO}_{4}$.
}

TABLE 4.6. Definition of Reference Codes for Tables 4.1 Through 4.5

\begin{tabular}{|c|c|c|c|}
\hline Code & Reference & Code & Reference \\
\hline $68 \mathrm{HPQ}$ & $\begin{array}{l}\text { Hingston, Posner, and } \\
\text { Quirk (1968) }\end{array}$ & $82 \mathrm{aBM}$ & $\begin{array}{l}\text { Balistrieri and Murray } \\
\text { (1982a) }\end{array}$ \\
\hline $\begin{array}{l}754 \\
78 B\end{array}$ & $\begin{array}{l}\text { Yates }(1975) \\
\text { Benjamin }(1978)\end{array}$ & $82 \mathrm{bBM}$ & $\begin{array}{l}\text { Balistrieri and Murray } \\
\text { (1982b) }\end{array}$ \\
\hline $780 \mathrm{~L}$ & Davis and Leckie (1978) & $85 \mathrm{HL}$ & Hsi and Langmuir (1985) \\
\hline $79 B M$ & $\begin{array}{l}\text { Balistrieri and Murray } \\
\text { (1979) }\end{array}$ & 85 SMS & $\begin{array}{l}\text { Sanchez, Murray and } \\
\text { Sibley (1985) }\end{array}$ \\
\hline $800 L$ & Davis and Leckie (1980) & $86 C L$ & Catts and Langmuir (1986) \\
\hline 8005 & Dempsey and Singer (1980) & $87 \mathrm{HaL}$ & Hayes and Leckie (1987) \\
\hline 8OLBHKA & Leckie et al. (1980) & 87 ZGSR & Zachara et al. (1987) \\
\hline $81 B B$ & Benjamin and Broom (1981) & B8AGZS (a) & Ainsworth et al. (1988) \\
\hline $81 B M$ & $\begin{array}{l}\text { Balistrieri and Murray } \\
\text { (1981) }\end{array}$ & 88 GASM $^{(b)}$ & Girvin et a 1. (1988) \\
\hline
\end{tabular}

(a) Ainsworth, C. C., D. C. Girvin, J. M. Zachara, and S. C. Smith. 1988. "CrOP. Adsorption on Goethite: Effects of Aluminum Substitution." Soil Sci. Soc. Am, J, in press.

(b) Girvin. D. C., L. L. Ames. A. P. Schwab, and J. E. McGarrah. 1988. "Neptunium Adsorption on Synthetic Amorphous Iron Oxyhydroxide." Interface Sci. in press. 


\subsection{EFFECTS OE SITE CONCENTRATION ON ACIDITY CONSTANTS AND D*K $\mathrm{K}^{i n t}$}

Acidity constants are calculated using Equation (3.6):

$$
K_{\mathrm{al}}^{\mathrm{int}}=\frac{\left[\mathrm{SOH}^{\mathrm{C}}\right]\left[\mathrm{H}^{+}\right]}{\left[\mathrm{SOH}_{2}^{+}\right]} \exp \left(\frac{-e \Psi_{0}}{\mathrm{kT}}\right)
$$

Equation (3.7):

$$
K_{\mathrm{a} 2}^{\text {int }}=\frac{[\mathrm{SO}]\left[\mathrm{H}^{+}\right]}{\left[\mathrm{SOH}^{0}\right]} \exp \left(\frac{-e \Psi_{0}}{\mathrm{KT}}\right)
$$

and the relationship:

$$
\left[\mathrm{SOH}^{0}\right]=\mathrm{SO}_{\text {total }}-\left[\mathrm{SO}^{-}\right]+\left[\mathrm{SO}_{2}^{+}\right]
$$

The acidity constants for Fe(III) hydrous axides (with the exception of those reported by Balistrieri and Murray 1981) are normally distributed with mean values of $5.0 \pm 0.5$ and $10.9 \pm 0.5$ for $p K_{a l}^{i n t}$ and $p k_{a 2}^{i n t}$. respectively (Table 4.1). No skewness is evident in the frequency distribution plot (not shown). Thus, within the resolution of these data, there is no difference between crystalline and amorphous solid structure (in terms of proton dissociation from hydroxylated iron sites).

As indicated by Equations (3.6), (3.7), and (4.1), the value of the acidity constant is dependent on the total number of sites ( $\mathrm{SO}_{\text {total }}$ ). The site density of an oxide is typically obtained from a surface area measurement of the solid by the BET method (Brunauer. Emmett, arid Teller 1938) and a determination of the number of binding sites by tritium exchange, acid/base titration, theoretical calculations, or differential capacitance measurements. These measurements allow an estimate of the total number of sites $\left(\mathrm{SO}_{\mathrm{total}}\right.$ ) per unit mass and per unit surface area of the oxide. By experimental design, [SO $\left.{ }^{-}\right]$and $\left[\mathrm{SOH}_{2}^{+}\right]$are determined directly by potentiometric titration, and [SOH ${ }^{\circ}$ ] is calculated from Equation (4.1). Therefore, variations in site density arising from differing experimental methads will be reflected in the values of acidity and complexation constants by variation in the calculated value of $\left[\mathrm{SOH}^{0}\right]$. The effects of these variations are illustrated by the two 
determinations of the surface properties of $\alpha$-FeOOH by Balistrieri and Murray (1979. 1981). The site density of 16.8 sites $\mathrm{nm}^{-2}$ used in the 1979 paper resulted in values of 4.9 and 10.4 for $p K_{a 1}^{\text {int }}$ and $p K_{a 2}^{\text {int }}$. respectively. The 1981 results, for material prepared under identical conditions and a reported site density of 2.6 sites $n m^{-2}$, are 5.6 and 9.5 for $\mathrm{pK}_{\mathrm{a} 1}^{\mathrm{int}}$ and $\mathrm{pK}_{\mathrm{a} 2}^{\mathrm{int}}$, respectively. The factor of seven difference between the two reported site densities accounts for the approximate factor of six difference between the two sets of acidity constants (Balistrieri and Murray 1981). The actual number of sites per mole of amorphous Fe(III) hydrous oxide or Mn(IV) oxide is likely to vary among preparations. However, based on the data tabulated here, it may be concluded that the differences in experimentally determined or assumed values of site density are a larger source of uncertainty than the actual variations. parallel differences will occur in the values of $p^{\star k^{i n t}}$ for specific ion adsorption because the $p^{\star} k^{i n t}$ values depend on the values of the acidity constants determined or assumed for the oxide.

Davis and Leckie (1978) have suggested that uncertainty in reported $p^{\star k^{i n t}}$ values can be minimized by writing the reactions in terms of surface complexation formation constants. $\log \mathrm{K}^{\mathrm{sc}}$ :

$$
\begin{gathered}
\mathrm{SO}+\operatorname{Me}(\mathrm{OH})_{n}^{2-n} \stackrel{K^{\mathrm{sc}}}{=} \mathrm{SO}^{-}-\mathrm{Me}(\mathrm{OH})_{n}^{2-n} \\
\log K_{\mathrm{Me}(\mathrm{OH})_{n}}^{\mathrm{sc}}=P K_{\mathrm{a} 2}^{\text {int }}-\mathrm{P}^{\star} K_{\text {me }(\mathrm{OH})_{n}}-\log \beta_{1 \pi}
\end{gathered}
$$

The use of $\log K^{s c}$, rather than $p^{\star} K^{i n t}$, removes the uncertainties associated with site density determinations. This is demonstrated by the data for copper adsorption onto amorphous fe(III) hydrous oxides. Values of 4.1

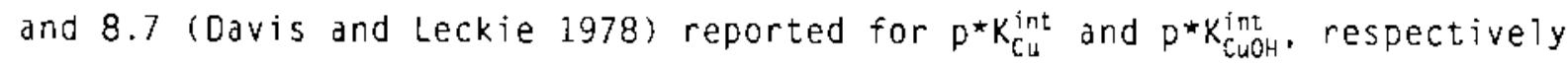
(Table 4.2) compare to values of 3.0 and 7.0 (Balistrieri and Murray 1982a) reported in Table 4.3. However, the values of $\log K_{C u}^{s c}$ and $l o g ~ K_{c u O H}^{s c}$ calculated for these values are of 6.6 and 9.9, respectively for the results of Davis and Leckie (1978) and 6.5 and 10.4 for Balistrieri and Murray (1982a). These results demonstrate that variations in the surface acidity constants arising from errors in site density are a significant source of uncertainty in the values of $p^{\star} k^{i n t}$ reported in the literature. 


\subsection{EFFECIS OF VARIABLE SITE OCCUPANCY ON $\mathrm{D} * K^{\text {int }}$}

Uncertainties in the numerical values of $p^{*} k^{i n t}$ arise from differences in the surface loading of the adsorbate on the oxide. Benjamin and Leckie (1981) report that, for most metal ions, fractional adsorption (i.e.. moles of metal adsorbed per mole of adsorbent in the system) decreases with increasing tota? metal concentration in a system with a fixed quaritity of adsorbent, even when surface complexation sites are available in excess, resulting in adsorbate concentration dependence of $p^{\star} k^{i n t}$. This observation is inconsistent with the assumption that all surface sites are energetically equivalent. However. Benjamin (1978) has demonstrated that at very low site coverage the most energetic adsorption site dominates the reaction, so that $p^{\star} k^{i n t}$ is independent. of adsorbate concentration. When the most energetic sites are filled, then the values of $p^{\star} k^{i n t}$ are dependent on the adsorbate concentration.

Sufficient data are available for $\mathrm{CdOH}^{+}$and $\mathrm{HCrO}_{4}^{-}$to evaluate the consequences of the progressive lodding of surface sites on log $k^{\text {sc }}$ values. Analyses of the results indicate that there significant correlations ( $r=$ 0.89 . Significant at the $1 \%$ level) between $\log K^{\text {sc }}$ and the adsorbate/adsorbent ratio (log Me total $\left./ \mathrm{Fe}_{\text {total }}\right)$. These results, shown in Figure 4.1a, yield the following regression equation:

$$
\log K_{\mathrm{CdOH}^{*}}^{\mathrm{sc}}=9.3-0.20 \log \left(\frac{M e_{\text {total }}}{F_{\text {total }}}\right)
$$

The values of $\log k^{\text {sc }}$ for some anions also show a marked dependence on concentrations of adsorbate and adsorbent in the system. This is exemplified in Figure $4.1 \mathrm{~b}$ for $\mathrm{HCrO}_{4}^{-}$. A significant correlation ( $r=0.90$, significant at the $1 \%$ level) between $\log \mathrm{K}^{\text {sc }}$ and the adsorbate/adsorbent ratio (log $\left.\mathrm{Me}_{\text {total }} / \mathrm{Fe}_{\text {total }}\right)$ is shown. In addition, $\log \mathrm{K}^{5 \mathrm{C}}$ for $\mathrm{HCrO}_{4}^{-}$is dependent on ( $\mathrm{r}=$ $-0.72)$ the value of $\log \mathrm{K}^{5 \mathrm{C}}$ used for $\mathrm{CrO}_{4}^{2-}$. Values for $\log \mathrm{K}^{\mathrm{sc}}$ for $\mathrm{HCrO}_{4}$ are given by: 

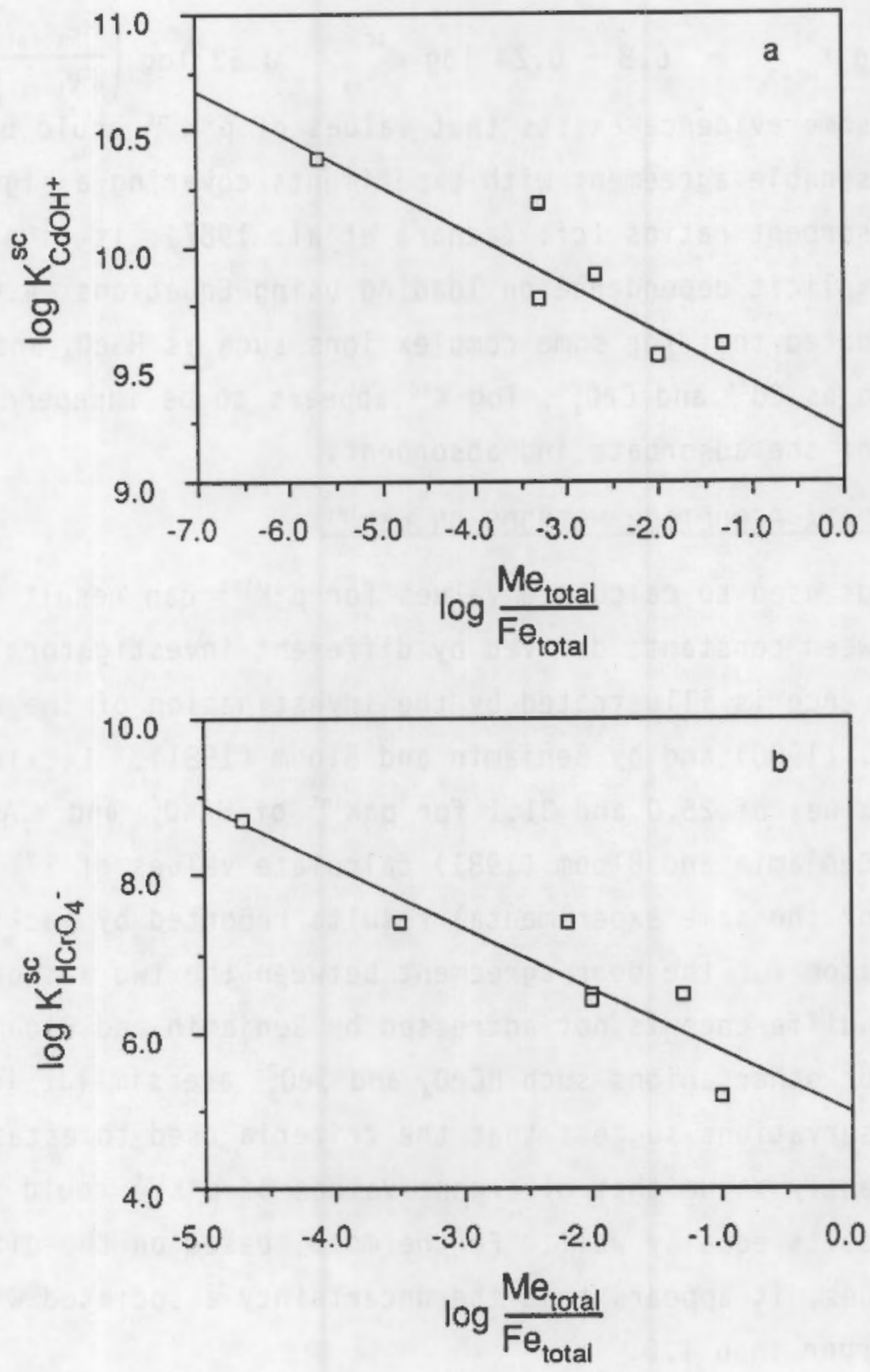

FIGURE 4.1. Dependence of (a) $\log \mathrm{K}_{\mathrm{CdOH}+}^{\mathrm{sc}}$ and (b) $\log \mathrm{K}_{\mathrm{HCrO4}}^{\mathrm{sc}}$. on the Experimental Adsorbate: Adsorbent Ratio 


$$
\log K_{\mathrm{HCrO}_{4}}^{\mathrm{sc}}=6.8 \cdot 0.24 \log K_{\mathrm{CrO}_{4}^{2-}}^{\mathrm{sc}}-0.63 \log \left(\frac{M e_{\text {total }}}{F_{\text {total }}}\right)
$$

Although some evidence exists that values of $p^{\star} K^{\text {int }}$ could be selected that are in reasonable agreement with experiments covering a significant range of adsorbate/adsorbent ratios (cf. Zachara et al. 1987). it is preferable to calculate the explicit dependence on loading using Equations (4.4) and (4.5). However, it is noted that for some complex ions such as $\mathrm{HSeO}_{4}^{-}$and $\mathrm{HSO}_{4}^{-}$and simple ions such as $\mathrm{Cd}^{2+}$ and $\mathrm{CrO}_{4}^{2-}, \log \mathrm{K}^{\text {sc }}$ appears to be independent of the concentrations of the adsorbate and absorbent.

\subsection{EFFECTS OF DATA REDUCTION METHODS ON $D * K^{\text {int }}$}

The methods used to calculate values for $D^{\star K^{i n t}}$ can result in significant differences between constants derived by different investigators. This potential difference is illustrated by the investigation of the As(V) system by Leckie et a1. (1980) and by Benjamin and Bloom (1981). Leckie et a1. (1980) report values of 25.0 and 31.1 for $\mathrm{P}^{\star K^{1 n t}}$ of $\mathrm{HASO}_{4}^{2-}$ and $\mathrm{H}_{2} \mathrm{AsO}_{4}^{-}$, respectively. Benjamin and Bloom (1981) calculate values of 27.7 and 33.5 . respectively, for the same experimental results reported by Leckie et al. (1980). The reason for the poor agreement between the two authors is not clear. and this difference is not addressed by Benjamin and Bloom. However. the constants for other anions such $\mathrm{HCrO}_{4}^{-}$and $\mathrm{SeO}_{3}^{2-}$ are similar in both data sets. These observations suggest that the criteria used to establish a best fit was sufficiently vague that divergent values of $p^{\star} k^{i n t}$ could fit the experimental results equally well. Furthermore, based on the difference between the values, it appears that the uncertainty associated with these $p \star k^{\text {int }}$ may be larger than 1.0 .

\subsection{EFFECTS OF ATMOSPHERIC CARBON DIOXIDE ON $\mathrm{D}^{\star} \mathrm{K}^{\text {int }}$}

Uncertainty in the values of $p * k^{i n t}$ for anions arises from the effects of carbonate adsorption. Zachara et a1. (1987) found that carbonate species arising from the dissociation of dissolved atmospheric $\mathrm{CO}_{2}$ were adsorbed and influenced $\mathrm{CrO}_{4}^{2-}$ adsorption via electrostatic interactions or competition for sites. Their results indicate that the introduction of atmospheric $\mathrm{CO}_{2}$ into anion adsorption experiments can significantly influence the results. They 
suggest that it should be treated as a variable in future experimental work with anions. In the case of cation adsorption. Benjamin and Bloom (1981) report that the competition between anions and cations for surface sites is relatively minor. Therefore, the effect of carbonate on anion adsorption is probably larger than on cation adsorption.

\subsection{EFFECTS OF INNER - SPHERE COMPLEX FORMATION ON $0^{\star} K^{\text {int }}$}

The TLM described in this report is based on the assumption that al 1 specifically adsorbing ions adsorb in the $\beta$-plane (i.e., outer-sphere complexes). As discussed in Section 3.0. recent work by Hayes and Leckie (1987) evaluating the ionic strength dependence of the adsorption of lead on $\alpha-F e 00 H$ indicates that lead adsorbs to the 0 -plane (i.e., forms inner-sphere complexes). Although the effects of inner-sphere complex formation couid not be evaluated from available values of $p * k^{i n t}$. Hayes and Leckie (1987) found that variations in ionic strength had no effect on lead adsorption. Furthermore, Hayes et a1. (1987) demonstrate by X-ray absorption spectroscopy that selenate forms weakly bonded outer-sphere complexes and selenite forms strongly bonded inner-sphere complexes with the surface of $a-F e 00 H$, again suggesting that some ions adsorb to the 0 -plane. As a result, the complexation of some strongly adsorbed ions is not properly represented by the TLM, introducing an additional uncertainty from an apparent ionic strength dependence in the values of $p^{\star} K^{i n t}$. 


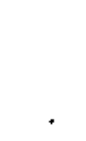




\subsection{EVALUATION OF TRIPLE-LAYER.PARAMETERS}

Approximately 130 values of acidity and intrinsic surface complexation constants for the TLM of oxide/water interfaces have been identified in the open literature and evaluated for amorphous Fe(III) hydrous oxide, $\alpha$-FeOOH, and $\delta-\mathrm{MnO}_{2}$. Values of $\log \mathrm{K}^{5 \mathrm{c}}$ calculated from values of $\mathrm{p}^{\star K^{i n t}}$ (Tables 4.2 through 4.5) and the hydrolysis constants given in Table 5.1 are tabulated in Tables 5.2 through 5.4. The selection of best values of $10 \mathrm{~g} k^{\text {sc }}$ was based on the average of several determinations or agreement with values calculated from regression equations given in Section 6.0. Uncertainties reported in this section are \pm 1 standard deviation.

\subsection{SURFACE ACIOITY CONSTANTS}

As reported in Section 4.1 , the acidity constant values for Fe(III) hydrous oxides are normally distributed with mean values of $5.0 \pm 0.5$ and 10.9 \pm 0.5 for $p K_{a l}^{i n t}$ and $p K_{a 2}^{i n t}$. respectively. Values of $\log k^{\text {sc }}$ for swamping electrolyte ions were calculated from the results presented in Table 4.1 and Equation (4.3). The average values of $\log \mathrm{K}^{\mathrm{sc}}$ were combined with the average acidity constants for fe(III) hydrous oxide to calculate $p^{\star} k^{i n t}$ values of $9.3 \pm$ 0.5 for $\mathrm{Na}^{+}$and $\mathrm{K}^{+}, 7.5 \pm 0.3$ for $\mathrm{NO}_{3}^{-}$, and $6.2 \pm 0.6$ for $\mathrm{Cl}^{-}$.

Two determinations of surface acidity constants for $\delta-\mathrm{MnO}_{2}$ are reported in Table 4.1. The differences between the values of $p k_{a z}^{i n t}$ for the two oxides are much larger than can be accounted for by differences in site density and may result from the use of materials with differing histories, although this does not seem likely given the similarities observed for Fe(III) hydrous oxides. The value of Catts and Langmuir (1986) is selected for this compilation because the measured values of $p^{\star k^{i n t}}$ for metals reported here are derived from their study.

\subsection{METALS AND METALLOIOS $D * K^{\text {int }}$}

Hydrolysis constants used in this study are presented in Table 5.1. Surface complexation constants (log $k^{s c}$ ) for cations and anions calculated using Equation (4.3) and the values of $p \star K^{i n t}, p K_{a 1}^{i n t}$. $p K_{a}^{i n t}$ reported in Tables 4.1 through 4.5 are given in Tables 5.2 through 5.4. Examination of Table 5.2 confirms the Davis and Leckie (1978) observation of similarities among values 
IABLE 5,1. Vaiues of $g_{1}, g_{2}$, and Ionic Radius ( $r$; for Cations (Brown, Sylva, and Ellis 1985) and Hydrolysis Constants (Baes and Mesmer 1976 and 1981)

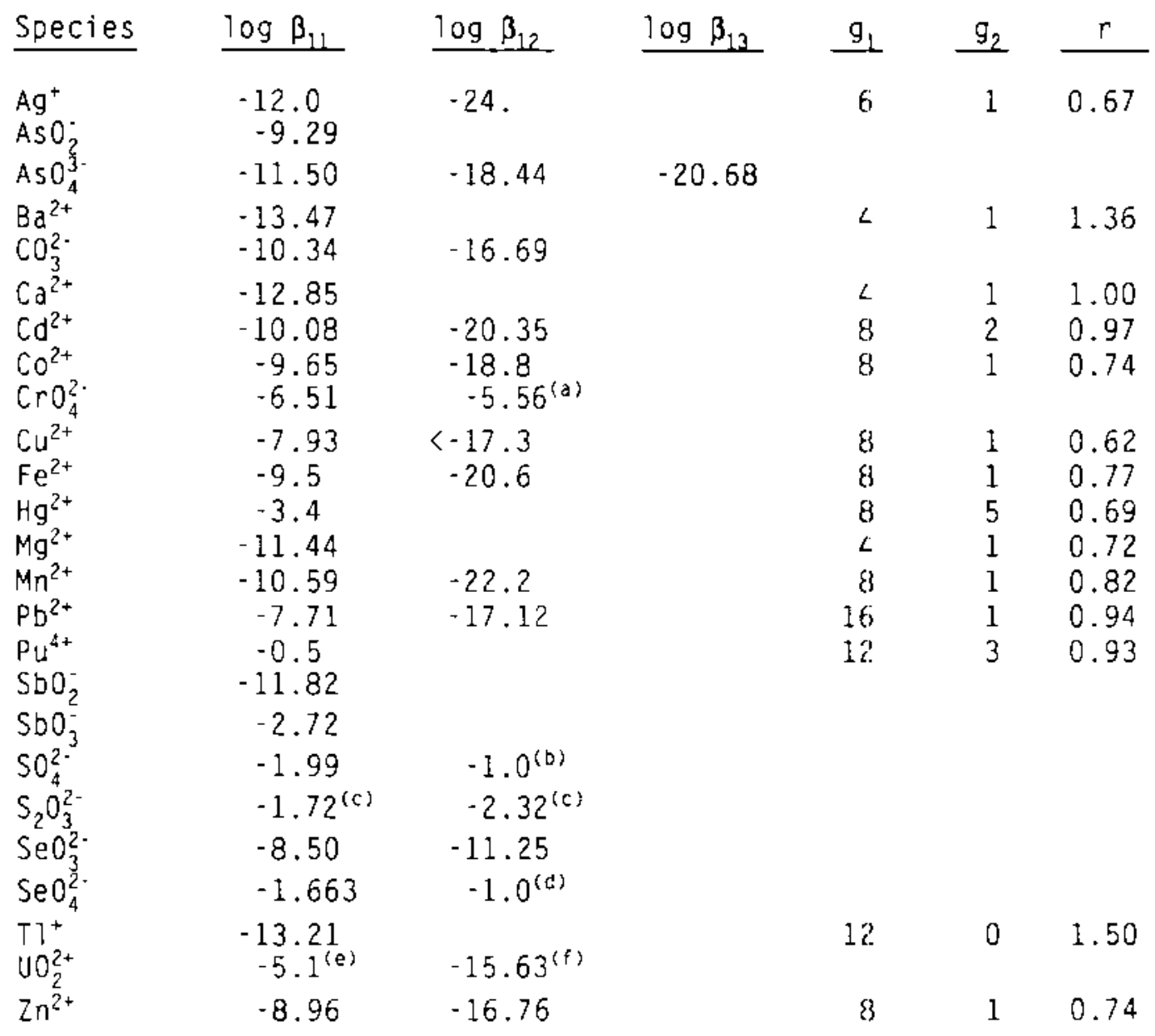

(a) Schmidt (1984).

(b) Truesdell and Jones (1974).

(d) Estimated.

(c) Smith. Popp, and Norman (1986).

(e) Dongarra and Langmuir (1980).

(f) $\log \beta_{35}$. 
TABLE 5.2. Surface Complexation Constants (log $k^{\text {sc }}$ ) for Cations on Fe(III) Hydrous oxide Substrates

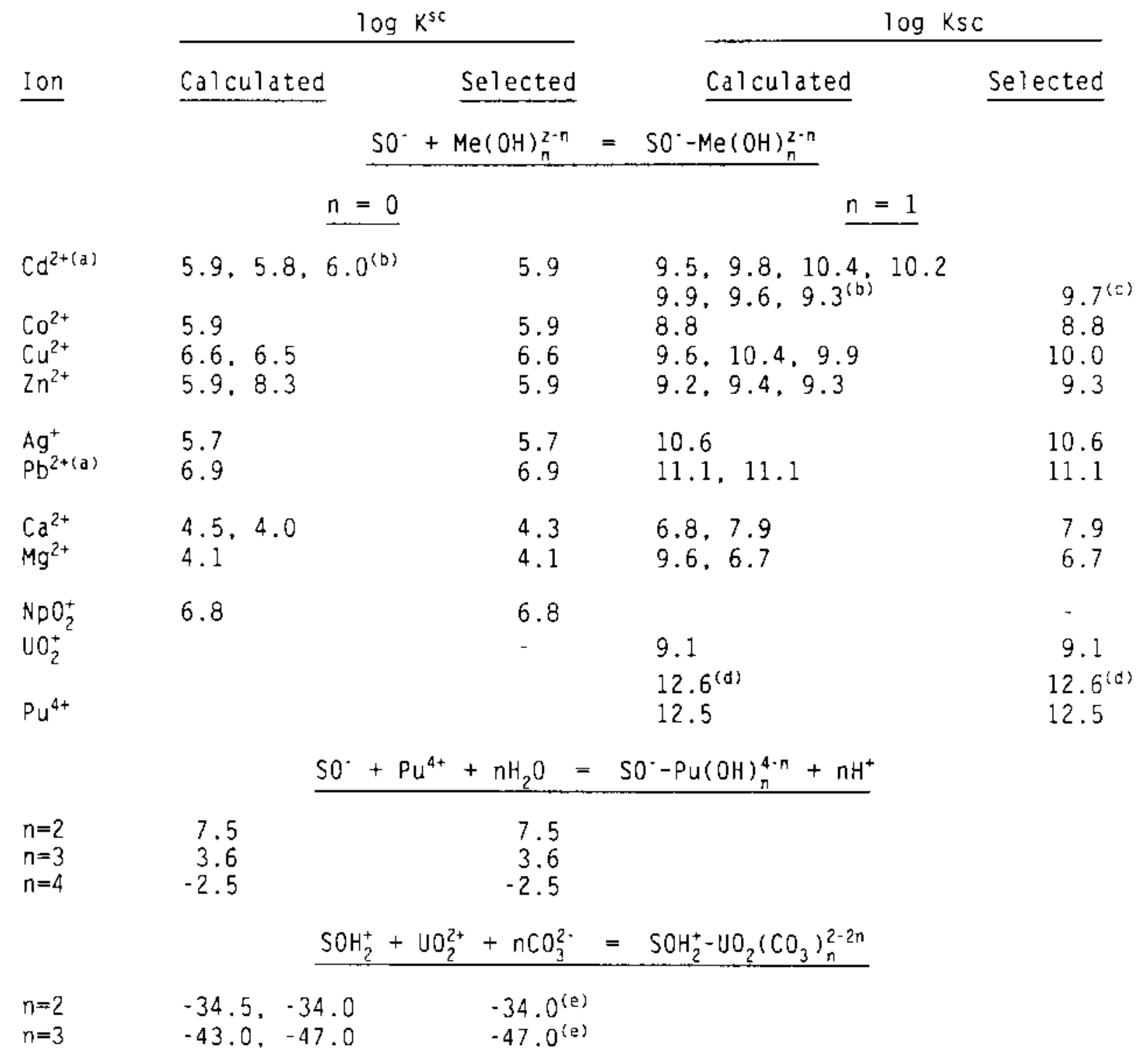

(a) Values of Balistrieri and Murray (1982b) are not included because the formation of aqueous chloride complexes was not treated adequately. (b) Oavis and Leckie (1978).

(c) Calculated from Equation (4.4).

(d) $\mathrm{SO}^{\circ}+\left(\mathrm{UO}_{2}\right)_{3}(\mathrm{OH})_{5}^{+}=\mathrm{SO}^{-}-\left(\mathrm{UO}_{2}\right)_{3}(\mathrm{OH})_{5}^{+}$.

(e) Selected value for $10^{-3}$ or less total carbonate. 
IABLE 5.3. Surface Complexation Constants ( $\log k^{\text {sc }}$ ) for Cations on Mn(IV) Oxide Substrates

I on

\begin{tabular}{|c|c|c|}
\hline$n=0$ & $\mathrm{n}=1$ & $n=2$ \\
\hline $\mathrm{SO}^{-}+$ & $-\pi$ & $\mathrm{SO}^{\circ}-\mathrm{Me}(\mathrm{OH})_{n}^{z \cdot \pi}$ \\
\hline
\end{tabular}

$\mathrm{Cu}^{2+}$

$2 n^{2+}$

6.1

6.6

10.1

$\mathrm{Pb}^{2+}$

4.7

6.4

8.0

$\mathrm{Ca}^{2+}$

$\mathrm{Mg}^{2+}$

8.0

7.4

0.9

0.3

IABLE 5.4. Surface Complexation Constants ( $10 \mathrm{~g} \mathrm{k}^{\mathrm{se}}$ ) for Anions on Fe(III) Hydrous Oxide Substrates

\begin{tabular}{|c|c|c|c|c|c|c|}
\hline \multirow[b]{2}{*}{ Ion } & \multicolumn{2}{|c|}{$\log k^{s c}$} & \multicolumn{2}{|c|}{$\log x^{5 c}$} & \multicolumn{2}{|l|}{$\log k^{s c}$} \\
\hline & Calculated & Selected & Calculated S & elected & Calculated & Selected \\
\hline & & $\mathrm{SOH}_{2}^{+}$ & $\mathrm{H}_{n} \mathrm{MeO}_{x}^{2+n \cdot 2 x}=\mathrm{SOH}$ & $-\mathrm{H}_{n} \mathrm{MeO}_{x}^{z+n \cdot 2 x}$ & & \\
\hline & $n=0$ & & $n=1$ & & $n=2$ & \\
\hline $\mathrm{AsO}_{4}^{3-}$ & & - & $9.3,8.4,11.1$ & 9.6 & $7.6 .7 .6,10.0$ & 8.4 \\
\hline $\mathrm{CO}_{3}^{2-}$ & & - & 5.0 & 5.0 & -2.1 & -2.1 \\
\hline $\mathrm{CrO}_{4}^{2-}$ & $\begin{array}{l}4.7,5.5,5.5 \\
6.8,9.3 .5 .6\end{array}$ & 5.6 & $\begin{array}{l}7.4,6.5,6.5 \\
6.4 .5 .2,8.7\end{array}$ & $6.7^{(0)}$ & & $\cdot$ \\
\hline $\mathrm{S}_{2} \mathrm{O}_{3}^{2-}$ & 4.9 & 4.9 & & - & & - \\
\hline $\mathrm{SO}_{4}^{2-}$ & $\begin{array}{l}6.2,6.2,4.8 \\
4.8\end{array}$ & 5.5 & $\begin{array}{l}9.9 \cdot 9.9,8.8 \\
8.8 .8 .8,9.5\end{array}$ & 9.2 & & 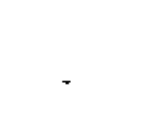 \\
\hline $\mathrm{SeO}_{4}^{2 \cdot}$ & $\begin{array}{l}4.8 .4 .8,6.7 . \\
9.9\end{array}$ & 5.5 & $\begin{array}{l}9.1 .9 .1,8.8 \\
12.2\end{array}$ & 9.2 & & - \\
\hline $\mathrm{SeO}_{3}^{2-}$ & $\begin{array}{l}7.4,6.3,7.7 . \\
7.8\end{array}$ & 7.3 & $\begin{array}{l}5.3 .4 .7 .7 .2 . \\
8.4\end{array}$ & $5.9^{(b)}$ & & - \\
\hline
\end{tabular}

(a) Calculated from Equation (4.5).

(b) Calculated from Equation (5.1). 
of $\log K^{s c}$. with values of $5.7 \pm 1.0$ and $9.7 \pm 1.4$ for $\log K_{M e}^{s c}$ and $\log K_{M e O H}^{s c}$. respectively. As noted before, although there are significant differences between values of $p^{*} K^{i n t}$ for copper and zinc determined by Benjamin and Bloom (1981); Davis, James, and Leckie (1978): and Balistrieri and Murray (1982b), the values for $\log k^{5 c}$ are similar. Selected best values for $\log k^{s c}$ are given in Tables 5.2 through 5.4 and combined with mean acidity constants and hydrolysis constants to provide recommended values of $p \star k^{\text {int }}$.

In many instances, only a single determination of $p^{\star k^{i n t}}$ for a given adsorbate/adsorbent system was found. As a result. statistical treatment of the data for all ions was not possible. Therefore, based on the result of regression analyses for a 11 cations, discussed in section 6.0 , uncertainties are estimated as \pm 0.5 for $\log K_{M e}^{s c}$ and \pm 0.8 for $\log K_{M e o H}^{s c}$. unless otherwise stated.

\subsubsection{Cation Adsorption on Fe(LIL) Hydrous Oxide}

Values of $\log k^{s c}$ for cation adsorption onto Fe(III) hydrous oxide are presented in Table 5.2. The choices of the selected values are described below.

\section{Cadnium}

Three $\log k_{C d}^{\text {sc }}$ values ranging from 5.8 to 6.0 were calculated, and the mean value of 5.9 is selected. As reported in Section 4.2 , the values of $10 \mathrm{~g}$ $\mathrm{K}_{\mathrm{C} \mathrm{dOH}}^{\mathrm{sc}}$ exhibit dependencies on the adsorbate: adsorbent ratio (Figure 4,1a). A value of $9.7 \pm 0.2$ was calculated for $\log k_{\text {cdoH }}^{s c}$ using Equation (4.4) for reference concentrations of $\mathrm{Me}_{\text {total }}=10^{-5}$ and $\mathrm{Fe}_{\text {total }}=10^{-3}$. The values determined in $\mathrm{NaCl}$ solutions by Balistrieri and Murray (1982b) are not included in the regression equation or Figure 4.1 a because the authors did not account for the formation of aqueous cadmium chloride complexes.

Cobalt

Values of 5.9 and 8.8 are reported for $\log K_{C O}^{5 c}$ and $\log K_{6 O O H}^{\mathrm{sc}}$. respectively. These values are selected.

\section{Copoer}

Two similar values $(6.5$ and 6.6$)$ are reported for $\log K_{C u}^{5 c}$, and the mean value of 6.6 is selected. Three values with a range of 9.6 to 10.4 are 
reported for $\log \mathrm{K}_{\mathrm{CuOH}}^{\mathrm{sc}}$. The mean value of 10.0 is selected.

Zinc

Two values ( 5.9 and 8.3 ) are reported for $\log \mathrm{K}_{2 \pi}^{5 \mathrm{c}}$. The value (8.3) of Dempsey and singer (1980) is much larger than values for other transition metals in Table 5.2 and is rejected. The selected value is 5.9 (Benjamin and Bloom 1981). Three values with a range of 9.2 to 9.4 are reported for $\log$ $K_{2 n C H}^{5 c}$. The mean value of 9.3 is selected.

Silver

Values of 5.7 and 10.6 are reported for $\log K_{A g}^{S C}$ and $\log K_{A g O H}^{S C}$. respectively. These values are selected.

\section{Lead}

The single value of 6.9 reported for $\log K_{p j}^{s i}$ is selected. Two identical values, 11.1 , are reported for $\log \mathrm{K}_{\mathrm{PDOH}}^{\mathrm{sc}}$. This value is selected. The values determined in NaCl solutions by Balistrieri and Nurray (1982b) are not included because they did not account for the formation of aqueous lead chloride complexes.

\section{Calcium}

Two values (4.0 and 4.5 ) are reported for $\log X_{C a}^{\text {sc }}$. The mean value of 4.3 is selected. Two values (6.8 and 7.9) are reported for $\log \mathrm{K}_{\text {CaOH }}^{\text {sc }}$. Both values were determined at different times by Balistrieri and Murray (1979, 1981). The surface complexes considered during data reduction were different between determinations. The 1979 determination considered the formation of a single $\left(\mathrm{SO}^{-}-\mathrm{CaOH}^{+}\right)$surface species. The 1981 detarmination considered the formation of an additional surface species $\left(\mathrm{SO}^{-}-\mathrm{Ca}^{2+}\right)$. Therefore, the two constants are not directly comparable. The 1981 value of 7.9 for $\log \mathrm{K}_{\mathrm{cacH}}^{\mathrm{sc}}$ is selected because both surface species (i.e.. SO $\mathrm{SO}^{-} \mathrm{CaOH}^{+}$and $\mathrm{SO}^{-}-\mathrm{Ca}^{2+}$ ) were considered.

\section{Magnesium}

The single value of 4.1 for $\log K_{M g}^{5 c}$ is recommended. Two values $(9.6$ and 6.7 ) are reported for $\log \mathrm{K}_{\mathrm{sg} \text { st }}^{\mathrm{se}}$. The value of 6.7 (Balistrieri and Murray. 1981 ) is selected for the same reason give for $\log \mathrm{K}_{\mathrm{CaOH}}^{5 \mathrm{C}}$.

\section{Actinides}

No duplicate determinations were found for $\log k^{\text {sc }}$ of actinide ions. 
The selected values are given in Table 5.2.

\section{2 .2 Cation Adserotion on Mn(IV) Oxide}

The only reported values for $p^{\star} k^{i n t}$ identified are those of Catts and Langmuir (1986) for copper, lead, and zinc and those of Balistrieri and Murray (1982a) for calcium, magnesium. and potassium. Again, values of log $\mathrm{K}^{\text {sc }}$ were calculated, and the calculated and selected values are presented in Table 5.3 .

\section{2 .3 Anion Adsorotion on Fe(III) Hydrous Oxides}

Data for anions are available primarily from Leckie et al. (1980) and Benjamin and Bloom (1981) and are presented in Table 4.5. In the case of As $(V)$, there are multiple $p \times K^{i n t}$ values derived from the same experiments by different investigators (Leckie et a1. 1980; Benjamin and B100m 1981). allowing an evaluation of the uncertainties associated with the reduction of experimental results. Furthermore, $p^{\star} k^{i n t}$ values calculated for most anions reported in Table 4.5 have been determined under varying experimental conditions, allowing evaluation of the effects of experimental conditions on the constants. Values of $l o g \mathrm{~K}^{\text {sc }}$ calculated for anions in Table 4.5 are given in Table 5.4 and discussed below.

\section{Arsenate}

Three values with a range of 9.3 to 11.1 are given for $\log K_{\text {HASO4 }}^{5 C}$. The mean value of $9.6 \pm 1.4$ is selected. Three values with a range of 7.6 to 10.0 are given for $\log \mathrm{K}_{\mathrm{H} 2 \mathrm{ASO} 4}^{\mathrm{SC}}$. The mean value of $8.4 \pm 1.4$ is selected.

\section{Carbonate}

Measured values of 5.0 and -2.1 (Zachara et al. 1987) for $\log \mathrm{K}_{\mathrm{Hcos}}^{\mathrm{sc}}$ and $\log K_{H 2 C O 3}^{5 C}$. respectively are selected. Values of 6.4 and 10.3 are calculated for $\log K_{H C O 3}^{\text {sc }}$ and $\log K_{c 03}^{s c}$ from the estimated $D^{\star} K^{i n t}$ reported by Sanchez. Murray, and Sibley (1985). The measured values of Zachara et al. (1987) are selected.

\section{Chromate}

Six values of $\log K_{\text {Crod }}^{s c}$ with a range of 4.7 to 9.3 are reported. Excluding the highest value (Benjamin and Bloom 1981), the mean and recommended value is $5.6 \pm 0.8$. As reported in Section 4.2 , the values of $\log$ $\mathrm{K}_{\mathrm{HCr}}^{5 \mathrm{C}} \mathrm{e}$ exhibit dependencies on the adsorbate:adsorbent ratio and the value of $\log K_{C \text { rOS }}^{5 c}$ (Figure $4.1 \mathrm{~b}$ ). A value of $6.7 \pm 0.6$ is calculated from Equation 
(4.5) for reference concentrations of $\mathrm{Me}_{\text {total }}=10^{-j}$ and $\mathrm{Fe}_{\text {total }}=10^{-3}$ and the selected value of log $K_{C r D A}^{s c}(T a b l e ~ 5.4)$.

\section{Thiosulfate}

A single value of 4.9 is reported for $\log K_{5203}^{i c}$. Based on sulfate and selenate, the uncertainty in this value is estimated as \pm 0.8 .

\section{Sulfate and Selenate}

Leckie et a 1. (1980) and Davis and Leckie (1980) report that the adsorption behavior of $\mathrm{SO}_{4}^{2-}$ and $\mathrm{SeO}_{4}^{2-}$ are identical. As a result, these ions are treated together here. Eight values with a range of 4.8 to 9.9 are reported for $\log \mathrm{K}_{\mathrm{Me} 04}^{\mathrm{sc}}$ (where $M e$ is $S$ or $S e$ ). Excluding the value of 9.9 (Benjamin and $8100 m 1981$ ) results in a mean value of $5.5 \pm 0.8$ for $\log \mathrm{K}_{\mathrm{Me} 0 \mathrm{sc}}^{\mathrm{sc}}$. Ten values with a range of 9.1 to 12.2 are reported for $10 g \mathrm{~K}_{\text {MMe04 }}^{\text {sc }}$. Excluding the value of 12.2 (Benjamin and Bloom 1981) results in a mean value of $9.2 \pm$ 0.5 for $\log \mathrm{K}_{\mathrm{HMEO} 4}^{\mathrm{sc}}$.

\section{Selenite}

Four values with a range of 6.3 to 7.8 are reported for $l o g K_{S e 03}^{s c}$. The mean value of $7.3 \pm 0.7$ is selected. The values of $\log \mathrm{K}_{\mathrm{HSeO}}^{\mathrm{sc}}$ exhibit dependency on the adsorbate:adsorbent ratio. Values for log $\mathrm{K}_{\mathrm{HSea}}^{\mathrm{sc}}$ are given by:

$$
\log K_{\mathrm{HSeO}_{3}^{5 c}}^{5 \mathrm{~s}}=-7.1+2.0 \log K_{\mathrm{SeO}_{3}^{2 .}}^{\mathrm{sc}}+0.68 \log \left(\frac{\text { Me }}{\mathrm{Fe}_{\text {total }}}\right)
$$

Using the selected value for $\log k_{\text {se03 }}^{\text {sc }}$ from Table 5.4. Equation (5.1) yields $\log \mathrm{K}_{\mathrm{HSe} 03}^{\mathrm{Sc}}$ of $5.9 \pm 1.4$ for reference conditions of $\mathrm{Me}_{\text {total }}=10^{-5}$ and $\mathrm{Fe}_{\text {total }}=$ $10^{-3}$.

\subsubsection{Anion Adsorption on Mn(IV) Oxide}

No values for anion adsorption on Mn(IV) oxide were found. Both Balistrieri and Murray (1982a) and Catts and Langmuir (1986) report that constants for $\mathrm{Cl}^{-}$, $\mathrm{SO}_{4}^{2-}$, and $\mathrm{NO}_{3}$ adsorption could not be determined because of the low (less than 2) $\mathrm{pH}$ of zero point charge $(Z \mathrm{PC})$. Therefore, anion adsorption on $\delta-\mathrm{MnO}_{2}$ is probably not as significant as adsorption on Fe(III) hydrous oxides. 


\subsection{PREDICTION OF $P \star K^{\text {int }}$}

A correlation between the intrinsic stability constants of surface complexes and corresponding values for cation hydrolysis has been recognized (Schindler et a 1. 1976). Figure 6.1 displays the relationship between values of $p^{\star} K^{i n t}$ (reported in Tables 4.2 through 4.5 ) and values of $-\log \beta_{1 n}$ (reported in Table 5.1) for cations and anions. Although general correlations are apparent the use of such plots to predict values of $p^{\star} K^{\text {int }}$ for which measured values are unavailable are of limited use because of the large uncertainties (1.4 to $2.0 \mathrm{log}$ units) associated with such estimates. Such correlations represent little advantage over using the mean values of $5.7 \pm 1.0$ and $9.7 \pm$ 1.4 for $\log \mathrm{K}_{\mathrm{Me}}^{\mathrm{sc}}$ and $\log \mathrm{K}_{\mathrm{MeOH}}^{\mathrm{sc}}$, respectively, derived from the results for Fe(III) hydrous oxides presented in Table 5.2 .

Baes and Mesmer (1981) derived expressions for estimating first hydrolysis constants for cations from the size-to-charge ratio. They found that the cations were described by four equations with the same slopes and different intercepts. Brown, Sylva, and Ellis (1985) found that if effective nuclear charge was used instead of simple charge that all the first hydrolysis constants for all cations could be described by a single expression:

$$
\begin{gathered}
\log \beta_{11}=\text { intercept }+ \text { slope }\left[g_{1}\left(\frac{z}{r^{2}}+g_{2}\right)\right] \\
g_{1}=(1+2 S+D)(z+2) \\
g_{2}=g(n)(z-1)+0.1 d(n-3)^{2}(1-S)
\end{gathered}
$$

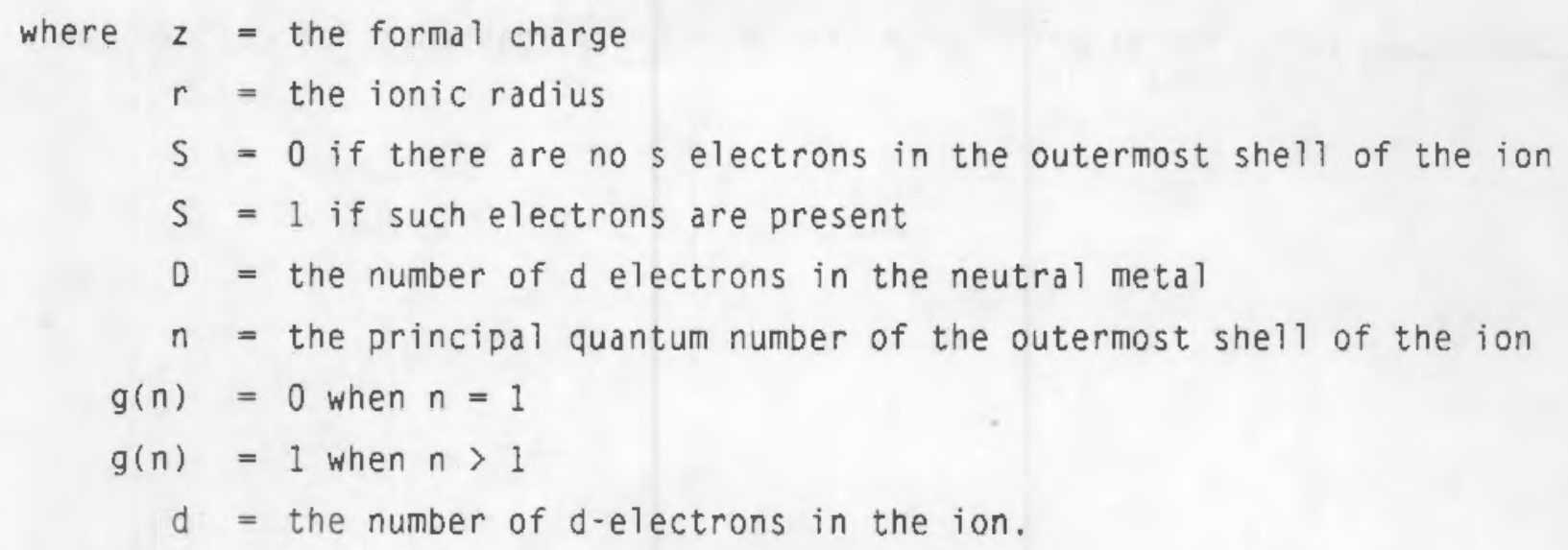



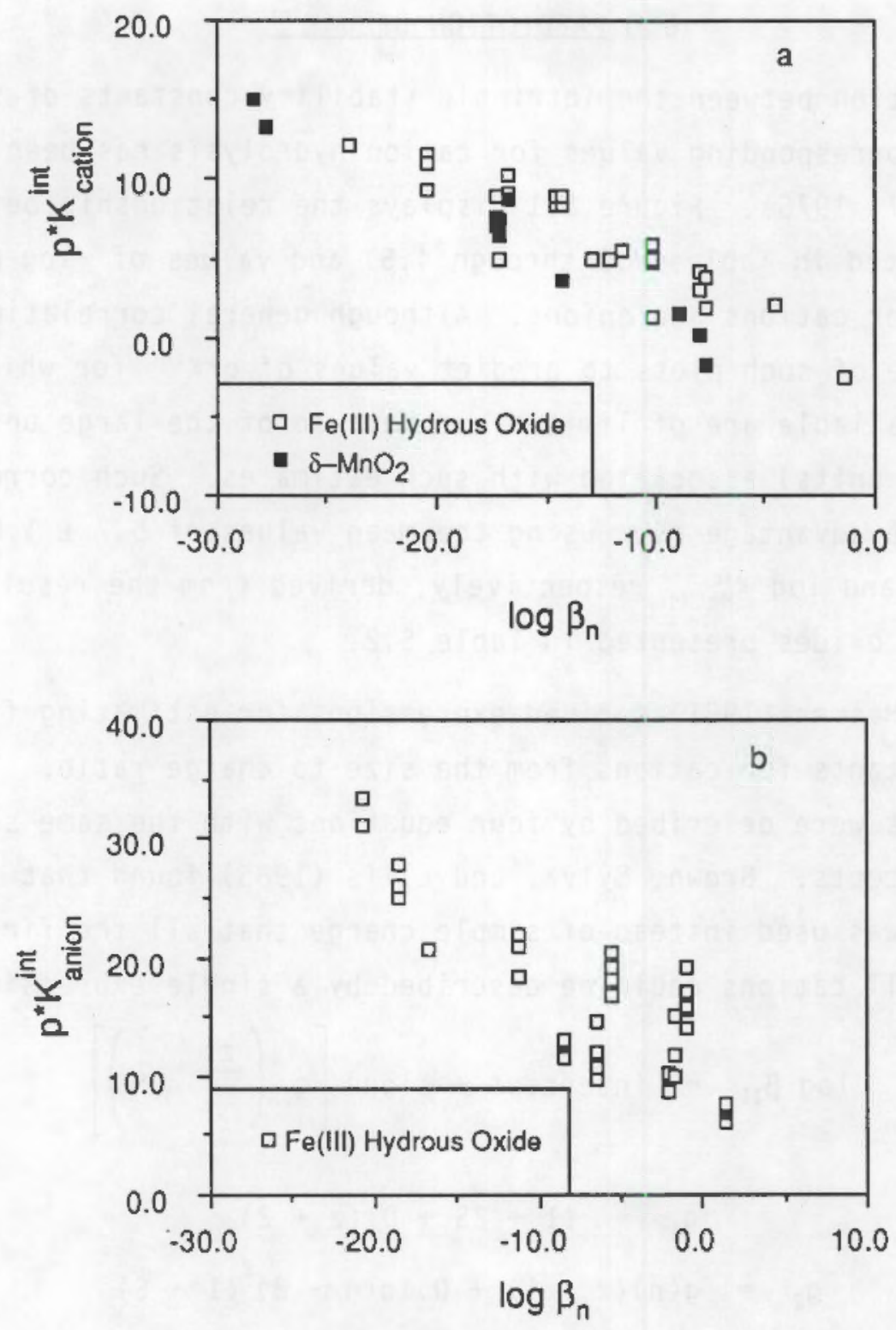

FIGURE 6.1. Variation of $p^{*} K^{\text {int }}$ with the Hydrolysis Constants for Cations (a) and Anions (b) 
The significance of the terms in Equations (6.1) through (6.3), as well as the derivations of these equations are given by Brown, Sylva, and Ellis (1985). Values of $g_{1}, g_{2}$, and $r$ for cations considered in this study are reported in Table 5.1.

The correlation observed between $D^{\star} K^{\text {int }}$ and $\log \beta_{1 n}$ is probably because of the dependency of both equilibrium constants on properties, such as charge and size of the aqueous ions. Using an expression of the same general form as Equation (6.1), values of $\log \mathrm{K}^{\mathrm{sc}}$ for cations can be calculated from:

$$
\log K_{\text {cation }}^{s c}=a_{0}+0.10\left[g_{1}\left(\frac{z}{r^{2}}+g_{2}\right)\right]+a_{1} \log \beta_{1 n}
$$

where $\log \beta_{1 n}$ is the nth aqueous hydrolysis constant for the surface complex (for SO-Me, $n=0$; SO-MeOH, $n=1$, etc., Table 5.1), and $a_{0}$ and $a_{1}$ are constants with values of 2.3 and $-0.37(r=0.95)$, respectively. for Fe(III) hydrous oxides and 0.9 and $-0.20(r=0.93)$ for $M n(I V)$ oxide. The first two terms on the right side of Equation (6.4) are equivalent to Equation (6.1). The third term allows the prediction of constants for hydrolysed surface species (i.e.. SO $-\mathrm{Me}(\mathrm{OH})_{n}$ ), as $\log \beta_{10}$ is 0 by convention. The value $(0.10)$ of the coefficient of the charge-to-size term in Equation (6.4) was found to be identical for both the $F e(I I I)$ and Mn(IV) system. This means that for a reaction of the type:

$$
\mathrm{FeO}^{-}-\mathrm{Me}^{2+}+\mathrm{MnO}^{-} \stackrel{\mathrm{K}^{\mathrm{ex}}}{=} \mathrm{FeO}+\mathrm{MnO}^{-}-\mathrm{Me}^{2+}
$$

$K^{\text {ex }}$ is independent of the cation considered. (a) If this observation were applied to all oxides, the limited number of $p^{\star} k^{\text {int }}$ for cations on $A$ (III) and Ti(IV) could be used to estimate values of $a_{0}$ and $a_{1}$ in Equation (6.4) and values of $\log \mathrm{K}^{\mathrm{sc}}$ estimated for cations for which no experimental determinations are available.

Equation (6.4) is depicted graphically in Figure 6.2a. Using Equation

(a) This is not equivalent to saying that the difference in adsorption between the two solids is independent of the cation considered. 

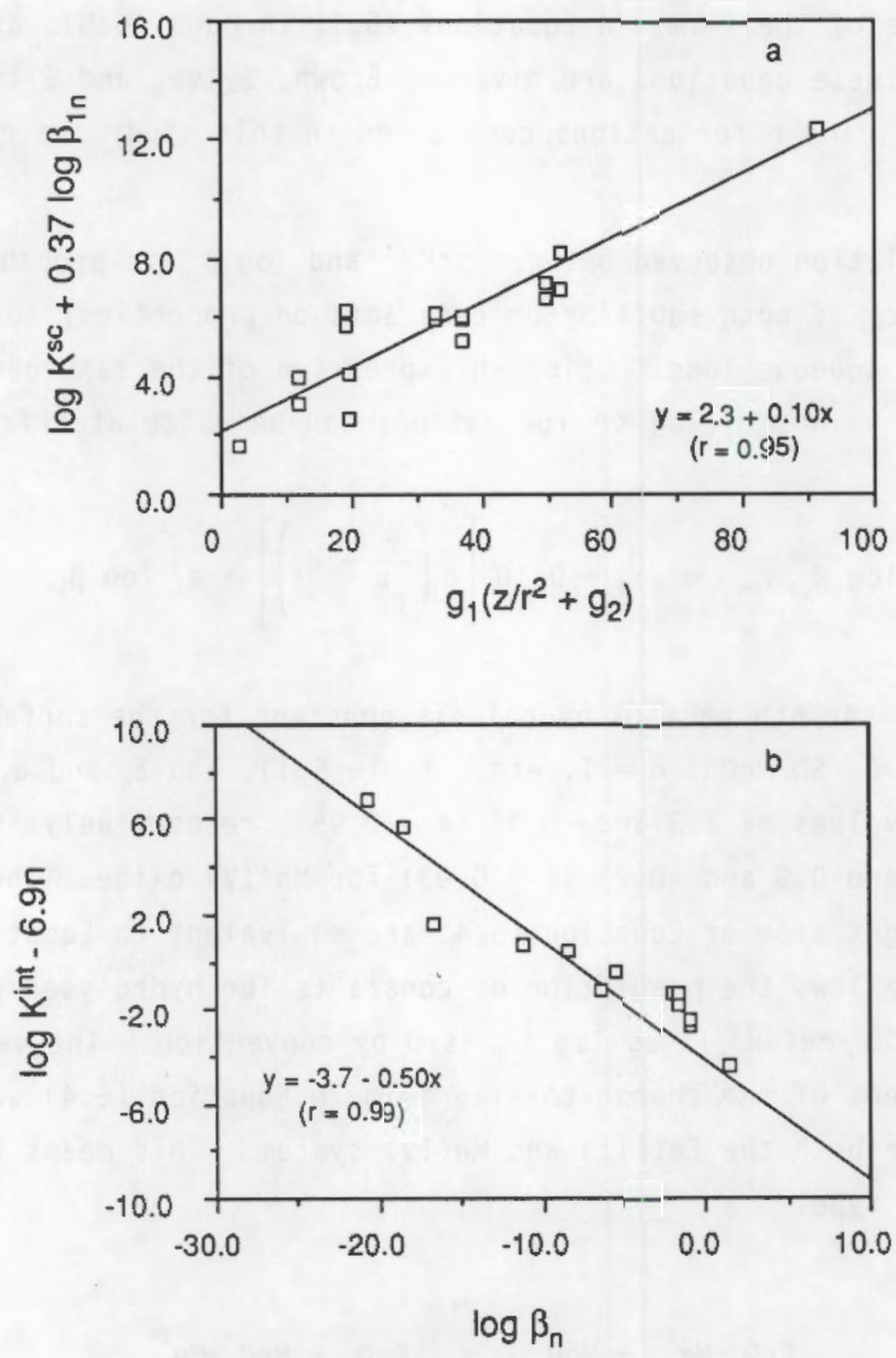

Figure 6.2. Representation of (a) Equation (6.4) and (b) Equation (6.8) for the Prediction of Surface Complexation Constants (log $K^{\text {sc }}$ ) for Cations on Fe(III) Hydrous Oxides and Intrinsic Surface Complexation Constants ( $l 0 g \mathrm{~K}^{\text {int }}$ ) for Anions on Fe(III) Hydrous Oxides 
(6.4) enables relatively accurate prediction of the values of $\log \mathrm{k}^{5 \mathrm{c}}$.

Excluding silver, the uncertainty associated with values estimated using this equation is \pm 0.5 for $\log \mathrm{K}_{\mathrm{Me}}^{\mathrm{sc}}$ and \pm 0.8 for $\log \mathrm{K}_{\mathrm{MeOH}}^{\mathrm{sc}}$. This uncertainty is comparable with the uncertainty in the measured values.

The values of $\log k^{s c}$ for silver complexes predicted by Equation (6.4) differ significantiy from the measured values of Davis and Leckie (1978). The reason for these differences is not currently clear. (a)

Attempts to correlate $\log k^{5 c}$ for anion adsorption with ion charge and size were not successful. This failure may be due in part to the large uncertainties associated with most values of $\log k^{\text {sc }}$ for anions. However. a good correlation $(r=0.99$. see Figure $6.2 \mathrm{~b})$ was obtained if $10 \mathrm{~g} k^{\text {int }}$ was used:

$$
\begin{gathered}
\mathrm{SOH}_{2}^{+}-\mathrm{H}_{n-1} \mathrm{MeO}_{\mathrm{m}}^{z+n \cdot 2 \mathrm{~m}-1} \stackrel{\mathrm{K}^{\text {int }}}{=} \mathrm{SOH}_{2}^{+}+\mathrm{MeO}_{\mathrm{m}}^{z-2 m}+(n-1) \mathrm{H}^{+} \\
\mathrm{H}_{n} \mathrm{MeO}_{\mathrm{m}}^{z+n-2 m} \stackrel{\beta_{n}}{=} \mathrm{nH}^{+}+\mathrm{MeO}_{m}^{z-2 m} \\
\log \mathrm{K}^{i n t}=-3.7-0.50 \log \beta_{n}+6.9 \mathrm{n}
\end{gathered}
$$

The standard error of estimate associated with Equation (6.8) is \pm 1.0 . which is consistent with the uncertainty in the measured values. The derivation of the coefficients in Equation (6.8) was accomplished using all the measured values (excluding outliers with residuals greater than three times the standard error) and not just the selected values.

From Equations (6.4) and (6.8). and the values of $\log \mathrm{k}^{\text {sc }}$ and $\log \beta$ : given in Table 5.1 through 5.4. it is possible to calculate or estimate values of $p^{\star} K^{i n t}$ for a large number of ions of interest in groundwater/sediment systems. Equations (6.4) and (6.8) can be combined with:

$$
P^{\star} K_{\text {cation }}^{i n t}=p K_{a 2}^{i n t}-\log K_{\text {cation }}^{\mathrm{sc}}-\log \beta_{1 \mathrm{n}}
$$

(a) Ozombzk (1986) suggests that the time allowed for equilibration by Davis and Leckie (1978) was not adequate in some cases. 


$$
p^{\star} K_{\text {anion }}^{\text {int }}=p K_{a 1}^{i n t}+\log K_{a n i o n}^{s c}-\log \beta_{n}
$$

and the values for surface acidity constants from Section 4.1 to yield:

$$
p^{\star} K_{\text {cation }}^{\text {int }}=8.6-0.63 \log \beta_{1 \mathrm{n}}-0.10\left[\mathrm{~g}_{1}\left(\frac{z}{r^{2}}+g_{2}\right)\right]
$$

for cation complexes with Fe(III) hydrous oxides and:

$$
p^{\star} K_{\text {cation }}^{\text {int }}=5.3-0.80 \log \beta_{1 n}+0.10\left[g_{I}\left(\frac{z}{r^{2}}+g_{2}\right)\right]
$$

for cation complexes with Mn(IV) oxides. Values of $p^{\star} k^{i n t}$ for anions are calculated from:

$$
p \times K_{\text {anion }}^{\text {int }}=1.3-0.50 \log \beta_{n}+6.9 n
$$

Recommended values of $p^{\star} K^{i n t}$ calculated from the selected values of $l o g$ $k^{5 c}$ (Tables 5.2, 5.3, and 5.4): the hydrolysis constants in Table 5.1: the surface acidity constants, presented in Section 4.1; and Equations (6.9) and (6.10) are presented in Table 6.1 for Fe(III) hyarous oxide and in Table 6.2 for Mn(IV) oxide. In addition, values of $p^{\star} k^{i n t}$ estimated using Equations (6.9) through (6.13) for anion and cations not ircluded in Tables 5.2 through 5.4 are also included in Tables 6.1 and 6.2 . Values of $p^{\star} k^{i n t}$ that are consistent with different acidity constants than used here can be calculated using Equations (6.9) and (6.10). In addition, values of $p^{\star} k^{\text {int }}$ for ions not considered in this study can be estimated from Equations (6.11), (6.12), and (6.13). The use of these estimates will facilitite initial efforts to model the behavior of pollutants and trace metals in groundwater/sediment systems. 
TABLE 6.1. Triple-Layer Model Intrinsic Complexation Constants for Fe(III) Hydrous Oxides Calcuated from Equations (6.9) and (6.10), or Calculated from Equations (6.11) and (6.13)

\begin{tabular}{|c|c|c|c|c|c|}
\hline Species & $p^{\pi} K^{i n t}$ & Species & $p^{\star} K^{i n t}$ & Species & $p^{*} k^{i n t}$ \\
\hline (a) $\mathrm{Ag}^{+}$ & 5.2 & (a) $\mathrm{Cu}^{2+}$ & 4.3 & (b), (e) $\mathrm{SbO}_{2}$ & (d) 14.1 \\
\hline (a) $\mathrm{AgOH}$ & 12.3 & (a) $\mathrm{CuOH}^{+}$ & 8.8 & (b) (f) $\mathrm{SbO}_{3}$ & (d) 9.6 \\
\hline (b) $\mathrm{HASO}_{4}^{2-}$ & 26.1 & (a) $\mathrm{Fe}^{2+}$ & (d) 5.1 & (b) $\mathrm{S}_{2} \mathrm{O}_{3}^{2-}$ & 9.9 \\
\hline (b) $\mathrm{H}_{2} \mathrm{ASO}_{4}^{-}$ & 31.8 & (a) $\mathrm{FeOH}^{+}$ & (d) 11.1 & (b) $\mathrm{SO}_{4}^{2-}$ & 10.5 \\
\hline (b). (c) $\mathrm{AsO}_{2}^{-}$ & (d) 12.9 & (a) $\mathrm{Hg}^{2+}$ & (d) 1.0 & (b) $\mathrm{HSO}_{4}$ & 16.2 \\
\hline (a) $\mathrm{Ba}^{2+}$ & (d) 7.8 & (a) $\mathrm{HgOH}^{+}$ & (d) 3.1 & (b) $\mathrm{SeO}_{4}^{2-}$ & 10.5 \\
\hline (a) $\mathrm{BaOH}$ & (d) 16.3 & (a) $\mathrm{Mg}^{2+}$ & 6.8 & (b) $\mathrm{HSeO}_{4}^{-}$ & 15.9 \\
\hline (b) $\mathrm{HCO}_{3}^{-}$ & 20.3 & (a) $\mathrm{MgOH}^{+}$ & 15.6 & (b) $\mathrm{SeO}_{3}^{2-}$ & 12.3 \\
\hline (b) $\mathrm{H}_{2} \mathrm{CO}_{3}^{\circ}$ & 19.6 & (a) $\mathrm{Mn}^{2+}$ & (d) 5.4 & (b) $\mathrm{HSeO}_{3}^{-}$ & 19.4 \\
\hline (a) $\mathrm{Ca}^{2+}$ & 6.6 & (a) $\mathrm{MnOH}^{+}$ & (d) 12.1 & (a) $\mathrm{Tl}^{+}$ & (d) 8.1 \\
\hline (a) $\mathrm{CaOH}^{+}$ & 15.9 & (a) $\mathrm{NpO}_{2}^{+}$ & 4.1 & (a) $\mathrm{T} 1(\mathrm{OH})^{\circ}$ & (d) 16.4 \\
\hline (a) $\mathrm{Cd}^{2+}$ & 5.0 & (a) $\mathrm{Pb}^{2+}$ & 4.0 & (b) $\mathrm{NO}_{2} \mathrm{OH}^{+}$ & 6.9 \\
\hline (a) $\mathrm{CdOH}^{+}$ & 11.3 & (a) $\mathrm{PbOH}^{+}$ & 7.5 & (a) $\left(\mathrm{UO}_{2}\right)_{3}(\mathrm{OH})_{5}^{+}$ & 13.9 \\
\hline (a) $\mathrm{CO}^{2+}$ & 5.0 & (a) $\mathrm{PuOH}{ }^{3+}$ & -1.1 & (g) $\mathrm{OO}_{2}\left(\mathrm{CO}_{3}\right)_{2}^{2-}$ & -29.0 \\
\hline${ }^{\text {(a) }} \mathrm{COOH}^{+}$ & 11.8 & (a) $\mathrm{Pu}(\mathrm{OH})_{2}^{2+}$ & 3.4 & (g) $\mathrm{NO}_{2}\left(\mathrm{CO}_{3}\right)_{3}^{4}$ & -42.0 \\
\hline (b) $\mathrm{CrO}_{4}^{2-}$ & 10.6 & (a) $\mathrm{Pu}(\mathrm{OH})_{3}^{+}$ & 7.3 & (a) $\mathrm{Zn}^{2+}$ & 5.0 \\
\hline (b) $\mathrm{HCrO}_{4}^{-}$ & 18.2 & (a) $\mathrm{Pu}(\mathrm{OH})_{4}^{\circ}$ & 13.4 & (a) $\mathrm{ZnOH}^{+}$ & 10.6 \\
\hline
\end{tabular}

(a) $\mathrm{SOH}^{\circ}+\mathrm{MMe}^{2+}+\mathrm{nH}_{2} \mathrm{O}=\mathrm{SO}^{-}-\mathrm{M}_{\mathrm{m}}(\mathrm{OH})_{n}^{2 \cdot \pi}+(\mathrm{n}+1) \mathrm{H}^{+}$.

(b) $\mathrm{SOH}_{2}^{+}-\mathrm{H}_{n} \mathrm{~A}^{\mathrm{n-Z}}=\mathrm{SOH}^{\circ}+\mathrm{A}^{2-}+(n+1) \mathrm{H}^{+}$.

(c) Equivelent to $\mathrm{As}(\mathrm{OH})_{4}$.

(d) Estimated.

(e) Equivelent to $\mathrm{Sb}(\mathrm{OH})_{4}$.

(f) Equivelent to $\mathrm{Sb}(\mathrm{OH})_{5}$ -

(g) $\mathrm{SOH}_{2}^{+}-\mathrm{UO}_{2}\left(\mathrm{CO}_{3}\right)_{n}^{2-2 \mathrm{n}}=\mathrm{SOH}^{\circ}+\mathrm{H}^{+}+\mathrm{UO}_{2}^{2+}+\mathrm{nCO}_{3}^{2-}$. 
IABLE 6.2. Triple-Layer Model Intrinsic Complexation Constants for $\delta \cdot \mathrm{MnO}_{2}$ Calcuated from Equation (6.9) or Equation (6.12)

\begin{tabular}{|c|c|c|c|c|c|}
\hline Species & $p^{\star K^{i \pi t}}$ & Species & $p^{\star} K^{i n:}$ & Species & $p^{\star K^{i n t}}$ \\
\hline (a) $\mathrm{Ag}^{+}$ & (b) 3.4 & (a) $\mathrm{Cu}^{2+}$ & 0.1 & (a) $\mathrm{MnOH}^{+}$ & (b) 10.6 \\
\hline (a) $\mathrm{AgOH}$ & (b) 13.0 & (a) $\mathrm{CuOH}^{+}$ & 7.5 & (a) $\mathrm{Mn}(\mathrm{OH})_{2}^{\circ}$ & (b) 19.9 \\
\hline (a) $\mathrm{Ba}^{2+}$ & (b) 4.5 & ${ }^{(a)} \mathrm{Cu}(\mathrm{OH})_{2}^{\circ}$ & 13.4 & (a) $\mathrm{Pb}^{2+}$ & -1.8 \\
\hline (a) $\mathrm{Ca}^{2+}$ & 5.3 & (a) $\mathrm{Fe}^{2+}$ & (b) 1.8 & (a) $\mathrm{PbOH}^{+}$ & 6.5 \\
\hline (a) $\mathrm{Cd}^{2+}$ & (s) 2.0 & (a) $\mathrm{FeOH}^{+}$ & (b) 9.4 & (a) $\mathrm{Tl}^{+}$ & (b) 4.8 \\
\hline (a) $\mathrm{CdOH}^{+}$ & (b) 10.1 & (a) $\mathrm{Fe}(\mathrm{OH})_{2}^{\circ}$ & (b) 18.3 & (a) $\mathrm{T} 1(\mathrm{OH})^{\circ}$ & (b) 15.3 \\
\hline${ }^{(a)} \mathrm{Cd}(\mathrm{OH})_{2}^{\circ}$ & (b) 18.3 & ${ }^{(a)} \mathrm{Hg}^{2+}$ & (b) -2.3 & (a) $\mathrm{Zn}^{2+}$ & 1.5 \\
\hline (a) $\mathrm{CO}^{2+}$ & (b) 1.6 & (a) $\mathrm{HgOH}^{+}$ & (b) 0.4 & (a) $\mathrm{ZnOH}^{+}$ & 8.8 \\
\hline (a) $\mathrm{COOH}^{+}$ & (b) 9.3 & (a) $\mathrm{Mg}^{2+}$ & 5.9 & (a) $\mathrm{Zn}(\mathrm{OH})_{2}^{\circ}$ & 15.0 \\
\hline (a) $\mathrm{CO}(\mathrm{OH})_{2}^{\circ}$ & (b) 16.6 & (a) $\mathrm{Mn}^{2+}$ & (b) 2.1 & & \\
\hline
\end{tabular}

(a) $\mathrm{SOH}^{\circ}+\mathrm{MMe}^{2+}+\mathrm{nH}_{2} \mathrm{O}=\mathrm{SO}^{\cdot}-\mathrm{M}_{m}(\mathrm{OH})_{n}^{2 \cdot n}+(\mathrm{n}+1) \mathrm{H}^{+}$.

(b) Estimated. 


\subsection{REFERENCES}

Atkinson, R. J.. A. M. Posner, and J. P. Ouirk. 1967. "Adsorption of Potential- Determining Ions at the Ferric Oxide-Aqueous Electrolyte Interface." J. Phys. Chem. 71:550-558.

Baes, C. F., Jr.. and R. E. Mesmer. 1976. The Hydrolysis of Cations. John Wiley and Sons, Inc.. New York.

Baes, C. F.. Jr., and R. E. Mesmer. 1981. "The Thermodynamics of Cations Hydrolysis." Am, J.Sci, 281:935-962.

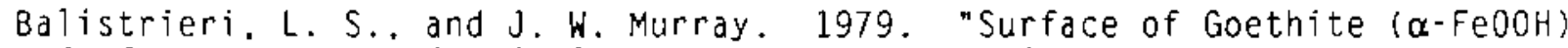
in Seawater." In Chemical Modeling in Aqueous Systems. ed. E. A. Jenne. No. 93, American Chemical Society Symposium Series, pp. 275-298.

Balistrieri. L. S.. and J. W. Murray. 1981. "The Surface Chemistry of Goethite $(\alpha-F e 00 H)$ in Major Ion Seawater." Am_l_sci. 281:788-806.

Balistrieri. L. S.. and J. W. Murray. 1982a. "The Adsorption of Cu, Pb, Zn, and Cd on Goethite from Major Ion Seawater." Geochim, Cosmochim, Acta $46: 1253-1265$.

Balistrieri, L. S., and J. W. Murray. 1982b. "The Surface Chemistry of $\delta-\mathrm{MnO}_{2}$ in Major Ion Seawater." Geochim. Cosmochim. Acta 46:1041-1052.

Barrow. N. J., and J. W. Bowden, 1987. "A Comparison of Models for Describing the Adsorption of Anions on a Variable Charge Surface. " Colloid Interface Sci, 119:236-250.

Benjamin. M. M. 1978. "Effects of Competing Metals and Complexing Ligands on Trace Metal Adsorption at the 0xide/Solution Interface." University Microfilms International. Ann Arbor. Michigan.

Benjamin. M. M., and N. S. Bloom. 1981. "Effects of Strong Binding of Anionic Adsorbates on Adsorption of Trace Metals on Amorphous Iron oxyhydroxide." In Adsoretion from Aqueous Solutions, ed. P. H. Tewari, Plenum, New York, pp. 41-59.

Benjamin. M. M., and J. 0. Leckie. 1981. "Multiple-site Adsorption of Cd. $\mathrm{Cu}, \mathrm{Zn}$. and $\mathrm{Pb}$ on Amorphous Iron 0xyhydroxide." J. Colloid Interface Sci. $79: 209-221$

Brown, P. L., R. N. Sylva, and J. Ellis. 1985. "An Equation for Predicting the Formation Constants of Hydroxo-metal Complexes." J. Chem. Soc Dalton Irans, $723-730$.

Brunauer. S., P. H. Emmett, and E. Teller. 1938. "Adsorption of Gases in Multimolecular Layers." J. Am. Chem. Soc. 60:309-319.

Catts, J. G.. and D. Langmuir. 1986. "Adsorption of Cu, Pb and $\mathrm{Zn}$ by $\delta-\mathrm{MnO}_{2}$ : Applicability of the Site binding Surface complexation Model." Applied Geochem. 1:255-264. 
Davis, J. A., and J. 0. Leckie. 1978. "Surface Ionization and Complexation at the $0 x i d e /$ water Interface. II. Surface Properties of Amorphous Iron oxyhydroxide and Adsorption of Metal Ions." J.Colloid Interface Sci. $67: 90-107$.

Davis, J. A., and J. 0. Leckie. 1980. "Surface Ionization and Complexation at the 0xide/water Interface. 3. Adsorption of Anions." l.Colleid Interface sci, $74: 32-43$.

Davis, J. A., R. 0. James, and J. 0. Leckie. 1978. "Surface Ionization and Complexation at the oxide/water Interface. I. Computation of Electrical Double Layer Properties in Simple Electrolytes." L.Colloid_Lnterface Sci. $63: 480-499$.

Dempsey, B. A., and P. C. Singer. 1980. "The Effects of Calcium on the Adsorption of Zinc by $\mathrm{MnO}_{x(s)}$ and $\mathrm{Fe}(\mathrm{OH})_{3(\mathrm{am})}$ " "n Contaminants and Sediments (vol. 2), ed. R. A. Baker. Ann Arbor Science, Ann Arbor, Michigan. pp. $334-352$.

Dongarra. G., and O. Langmuir. 1980. "The Stability of $\mathrm{UO}_{2} \mathrm{OH}^{+}$and $\mathrm{UO}_{2}\left(\mathrm{HPO}_{4}\right)_{2}^{2 .}$ Complexes at $25^{\circ} \mathrm{C}$." Geochim. Cosmochim. Acta $44: 1747-1751$.

Dzombak, D. A., and F. M. M. More1. 1985. "Adsorption of Inorganic Contaminants in Ponded Effluents From Coal-Fired Power Plants." Energy Lab Report No. MIT-EL 85-005. Cambridge. Massachusetts.

Dzombak. D. A. 1986. Toward a Uniform Model for the Sorption of Inorganic Ions on Hydrous oxides. Ph.D. Dissertation. Massachusetts Institute of Technology, Cambridge. Massachusetts.

Goldberg. S.. and G. Sposito. 1984. "A Chemical Model of Phosphate Adsorption by Soils: I1. Noncalcareous Soils." Soil Sci. Soc. Amer. Proc. 48: $779 \cdot 783$.

Hayes, X. M., and J. 0. Leckie. 1987. Modeling Ionic Strength Effects on Cation Adsorption at Hydrous 0xide/Solution Interfaces." I. Colloid Interface Sci, 115:564-572.

Hayes, K. M.. A. L. Roe, G. E. Brown, Jr.. X. O. Hodgson, J. O. Leckie, and G. A. Parks. 1987. "In Situ X-ray Adsorption Study of Surface Complexes: Selenium oxyanions on $\alpha$-Fe00H." Science 238:783-786.

Hingston, F. J., A. M. Posner, and J. P. Ouirk. 1968. "Adsorption of Selenite by Goethite." In Advance in Chemistry Series. 79, ed. R. J. Gourd. pp. $82-90$.

Hsi, C. - X. D.. and D. Langmuir. 1985. "Adsorption of Uranyl onto Ferric Oxyhydroxides: Application of the Surface Complexation Site-Binding Model." Geochim. Cosmochim. Acta 49:1931+1941.

Jenne. E. A. 1977. "Trace Element Sorption by Sediments and Soils-Sites and Processes." In Symposium on Molybdenum in the Environment vol. 2, eds W. Chappell and $k$. Peterson. M. Dekker, Inc.. New York.

pp. $425-553$. 
Jenne, E. A., D. M. DiToro, H. E. Allen, and C. Z. Zarba. 1986. "An Activity-Based Model for Developing Sediment Criteria for Metals: Part I. A New Approach." In Chemicals in the Environment, eds. J. N. Lester, R. Perry and R. M. Sterritt. pp. 560-568.

Leckie, J. 0.. M. M. Benjamin, K. Hayes, G. Kaufman, and S. Aitmann. 1980. "Adsorption/Coprecipitation of Trace Elements from Water with Iron Dxyhydroxide." CS+1513. Electric Power Research Institute, Palo Alto. California.

Minelcic. J, R. and R. G. Luthy. 1986. "Adsorption of Lead and Zinc on Blast Furnace Iron Oxide Solids." l. Water Poll. Control Fed. 58:242-249.

Morel, F. M. M., J.C. Hestal1, and J. G. Yeasted. 1981. "Adsorption Models: A Mathematical Analysis in the Framework of General Equilibrium Calculations." In Adsorption of Inorganics at Solid-Liquid Interfaces. eds. M. A. Anderson and A. J. Rubin. Ann Arbor Science, Ann Arbor. Michigan. pp. 263-294.

Morgan, J. J., and W. Stumm. 1964. "Colloidal Chemical Properties of Manganese Dioxide." J. Colleid Interface Sci. 19:347-359.

Murray. J. W. 1974. "The Surface Chemistry of Hydrous Manganese Dioxide." J. Colloid. Interface Sci, 46:357-371.

Sanchez. A. L.. J.W. Murray, and T. H. Sibley. 1985. "The Adsorption of Plutonium IV and $V$ on Goethite." Geochim, Cosmochim. Acta 49:2297-2307.

Schindler. P. W., B. Fürst, R. Dick, and P. U. Wolf. 1976. "Ligand Properties of Surface Silanol Groups. I. Surface Complex Formation with $\mathrm{Fe}^{3+}, \mathrm{Cu}^{2+}, \mathrm{Cd}^{2+}$, and $\mathrm{Pb}^{2+} . " \mathrm{l}$. Colleid Interface Sci, 55:469-475.

Schmidt. R. L. 1984. Thermodynamic Properties and Environmental Chemistry of Chromium. PNL-4881. Pacific Northwest Laboratory, Richland. Washington.

Smith. R.W., C. J. Popp, and D. I. Norman. 1986. "The Dissociation of 0xy-Acids at Elevated Temperatures." Geochim. Cosmochim. Acta 50:137-142.

Sposito. G. 1984. "Chemical Models of Inorganic Pollutants in Soils." CRC Crit. Rev. Environ. Control. 15:1-24.

Tessier. A., F. Rapin, and R. Carignan. 1985. "Trace Metals in Oxic Lake Sediments: Possible Adsorption onto Iron 0xides." Geochim. Cosmochim. Acta $49: 183-194$.

Truesde11. A.H.. and B. F. Jones, 1974. "WATEQ. A Computer Program for Calculating Chemical Equilibria of Natural Waters." J._Research US Geol. Surv. 2:233-348.

Yates, D. E.. S. Levine, and T. W. Healy. 1974. "Site-Binding Model of the Electrical Double Layer at the 0xide/water Interface." J. Chem. Soc. Faraday Trans. 1 70:1807-1818.

Yates. D. E. 1975. "The Structure of the Oxide/Aqueous Electrolyte Interface." Ph. D. Dissertation. University of Melbourne. Melbourne. Victoria, Australia. 
Zachara. J. M.. D. C. Girvin. R. L. Schmidt, and C. T. Resch. 1987.

"Chromate Adsorption on Amorphous Iron 0xyhydroxide in the Presence of Major Groundwater Ions." Environ. Sci. Tehnol. 21:589-594. 
APPENDIX A

SUMMARY OF EXPERIMENTAL CONDJIIONS 
APPENDIX A

SUMMARY OF EXPERIMENTAL CONDITIONS

\section{A.l Ainsworth et al. $(1988)^{\text {(a) }}$}

Goethite was prepared by the titration of $\mathrm{FeCl}_{3}$ with $\mathrm{CO}_{2}$-free $\mathrm{NaOH}$ (L-Fe) and by air oxidation of neutralized $\mathrm{FeCl}_{2}$ solutions (M-Fe). Material was characterized by $X$-ray diffraction. Surface areas of 33.7 and $176 \mathrm{~m}^{2} \mathrm{~g}^{-1}$ were determined by the $\mathrm{N}_{2}$ BET method for $L-F e$ and $M-F e$. respectively. Goethite was allowed to rehydrate in 0.01 or $0.1 \mathrm{M} \mathrm{NaNO}_{3}$, after which the $\mathrm{pH}$ was adjusted to 10.0 to 10.5 . After 2 hours the adsorbate was added, and 4 hours were allowed for equilibration. The acidity constants calculated by Davis and Leckie (1978) based on the data of Yates (1975) were used. Site densities of 16.4 (L-Fe) and 21.6 (M-Fe) sites $\mathrm{nm}^{-2}$ based on Yates (1975). and model calculations were used.

\section{A.2 Balistrieri and Murray (1979)}

Goethite was synthesized by the method of Atkinson. Posner. and Quirk (1967). The material was characterized by X-ray diffraction. A surface area of $48.5 \mathrm{~m}^{2} \mathrm{~g}^{-1}$ was determined by the $\mathrm{N}_{2}$ BET method. The surface-site density (16.8 sites $n m^{2}$ ) was from the tritium exchange experiments of Yates (1975).

\section{A. 3 Balistrieri and Murray (1981)}

The preparation and characterization of goethite was the same as reported by Balistrieri and Murray (1979). except the surface area was $51.8 \mathrm{~m}^{2}$ $\mathrm{g}^{-1}$. and surface-site density of 2.6 sites $\mathrm{nm}^{-2}$ was determined by titration in $1 \mathrm{M} \mathrm{NaCl}$ to a $\mathrm{pH}$ of 11 . Potentiometric titrations of the solid in $\mathrm{KCl}$ and $\mathrm{NaCl}$ with 2-minute equilibration times provide the data for the determination of the acidity constants. Intrinsic constants for $\mathrm{Ca}^{2+}$. $\mathrm{Mg}^{2+}$, and $\mathrm{SO}_{4}{ }^{2-}$ were determined from 24 -hour experiments.

\section{A.4 Balistrieri and Murray (1982a)}

The $\delta-\mathrm{MnO}_{2}$ was prepared by the method of Morgan and Stumm (1964) and characterized by X-ray diffraction. A surface area of $74 \mathrm{~m}^{2} \mathrm{~g}^{-1}$ was determined by the $\mathrm{N}_{2}$ BET method. The number of surface sites $\left(27.2\right.$ mole $\mathrm{kg}^{-1}$ ) was

\footnotetext{
(a) Ainsworth. C. C., D. C. Girvin. J.M. Zachara, and S. C. Smith. in press. "CrO ${ }_{4}^{2 .}$ Adsorption on Goethite: Effects of Aluminum Substitution." Soil Sci. Sec. Am, J, in press.
} 
determined from tritium exchange experiments. Their reported surface-site density (221 sites $\mathrm{nm}^{2}$ ) is much larger than for other oxides (such as Fe(III) hydrous oxides. $\mathrm{AI}_{2} \mathrm{O}_{3}, \mathrm{TiO}_{2}$, and $\mathrm{SiO}_{2}$ ) and is much larger than the value of 18 sites $\mathrm{nm}^{-2}$ determined for $\delta-\mathrm{MnO}_{2}$ by Catts and Langmuir (1986). Baristrieri and Murray (1982a) defend their surface-site density as being correct based on the agreement between their determination for $\alpha-\mathrm{FeOOH}$ and that of Yates (1975). Potentiometric titrations were conducted in $\mathrm{NaCl}$ solutions with 2. minute equilibration times. Adsorption of $\mathrm{Ca}^{2+}$ and $\mathrm{Mg}^{2+}$ was determined in $\mathrm{NaCl} / \mathrm{KCl}$ solutions equilibrated for 1 hour.

\section{A.5 Balistrieri and Murray (1982b)}

The materials, characterizations, and oxide triple-iayer parameters are the same as reported by Balistrieri and Murray (1981). Adsorption experiments for $\mathrm{Cd}, \mathrm{Cu}, \mathrm{Pb}$, and $\mathrm{Zn}$ were conducted in $0.1 \mathrm{M} \mathrm{NaNO}_{3}$ and equilibrated for 2.5 hours. However, no intrinsic constants were determined for these experiments. The reported results are based on experiments in $0.53 \mathrm{M} \mathrm{NaCl}$. Although the equilibration time is not specified, it may be the same as given above. Balistrieri and Murray found no difference in adsorption between experiments in $\mathrm{NaNO}_{3}$ and $\mathrm{NaCl}$, even though the solution activities of $\mathrm{Cd}^{2+}$ and $\mathrm{Pb}^{2+}$ are significantiy lower (because of the formation of chloride complexes) in the $\mathrm{NaCl}$ solution. They modeled the results using acuo ion stoichiometry rather than allowing for the formation of metal chloride surface complexes. This approach requires larger complexation constants for $\mathrm{Cd}^{2+}$ and $\mathrm{Pb}^{2+}$ in $\mathrm{NaCl}$ solutions relative to $\mathrm{NaNO}_{3}$ solutions. Their results for these two metals are not consistent with other determinations: therefore, they were not used.

\section{A.6 Benjamin (1978)}

Amorphous fe(III) hydrous oxide was prepared and characterized as described by Davis and Leckie (1978) except the precipitate was aged for 3 hours at $\mathrm{pH} 7$ and for 1 hour at the lowest pH used in the adsorption experiment. Adsorption experiments were conducted in $0.1 \mathrm{M} \mathrm{NaNO}_{3}$ with equilibration times of 4 hours. Surface properties of the oxide were apparently taken from Davis and Leckie (1978).

\section{A.7 Benjamin and B100m (1981)}

Amorphous fe(III) hydrous oxide was prepared and characterized as described by Davis and Leckie (1978) except the precipitate was aged for 2

\section{A. 2}


hours at $\mathrm{pH} 7$. Adsorption experiments were conducted in $0.1 \mathrm{M} \mathrm{NaNO}_{3}$ with equilibration times of 2 hours. Surface properties of the oxide were taken from Davis and Leckie (1978).

\section{A. 8 Catts and Langmuir (1986)}

The $\delta$-Mn02 was prepared by the method of Murray (1974) and characterized by $x$-ray diffraction. A surface area of $290 \mathrm{~m}^{2} \mathrm{~g}^{-1}$ was determined by the $\mathrm{N}_{2}$ BET method. Potentiometric titrations in $\mathrm{NaNO}_{3}$ with equilibration times of approximateiy 2 minutes were used to determine the acidity constants. Equilibration times of 3 days (with at least 1 hour per day on an automatic shaker) were used for $\mathrm{Cu}^{2+}, \mathrm{Pb}^{2+}$, and $2 \mathrm{n}^{2+}$ adsorption experiments. Site density (18 site $\mathrm{nm}^{-2}$ ) apparently was used as a fitting parameter, and the value was calculated from the titration data and the triple-layer model. Catts and Langmuir found that the constants derived could not adequately reproduce independent experiments when the adsorbate:adsorbent ratio of $10^{-3.9}$ was decreased by a factor of ten.

\section{A.9 Davis and Leckie (1978 and 1980$)$}

Amorphous fe(III) hydrous oxide was prepared in batch for each adsorption experiment from the titration of $\mathrm{Fe}\left(\mathrm{NO}_{3}\right)_{3}$ solution with $\mathrm{CO}_{2}$ free $\mathrm{NaOH}$. The precipitate was aged for 4 hours at a $\mathrm{pH}$ of 8 . Surface area of freeze-dried material as determined by the $\mathrm{N}_{2}$ BET method was $182 \mathrm{~m}^{2} \mathrm{~g}^{-2}$. However, in data reduction the surface area was treated as an adjustable parameter, and the value determined was $600 \mathrm{~m}^{2} \mathrm{~g}^{-1}$. The value for total sites $\left(9.85 \times 10^{-3} \mathrm{~mole}^{-1}\right)$ was determined by tritium exchange (Yates 1975) and recalculated by Davis and Leckie. These values lead to a surface-site density of 9.9 sites $\mathrm{nm}^{-2}$. Equilibration times (based on kinetic experiments) for metal adsorption experiments conducted in $0.1 \mathrm{M} \mathrm{NaNO}_{3}$ were 1 hour for anions. 2 hours for $\mathrm{Cu}$, and 4 hours for $\mathrm{Ag}$.

\section{A.10 Dempsey and Singer (1980)}

Amorphous Fe(III) hydrous oxide was prepared as described by Davis and Leckie (1978) except the precipitate was aged for 4 hours at pH 8.5. The surface area was assumed at $600 \mathrm{~m}^{2} \mathrm{~g}^{-1}$ based on Davis and Leckie (1978). Adsorption experiments were conducted with equilibration times of 16 to 18 nours. 


\section{A.11 Girvin et al. (1988) (a)}

Amorphous Fe(III) hydrous oxide was prepared as described by Davis and Leckie (1978). The material was aged up to 24 hours at unspecified pH. The surface parameters were determined from potentiometric titrations in $\mathrm{NaNO}_{3}$. The surface area of $600 \mathrm{~m}^{2} \mathrm{~g}^{-1}$ was obtained by mocel optimization and is identical to the value reported by Davis and Leckie (1978). The value for total sites $\left(9.85 \times 10^{-3}\right.$ mole $\left.\mathrm{g}^{-1}\right)$ was taken from Davis and Leckie (1978). Adsorption experiments were conducted in $0.1 \mathrm{M} \mathrm{NaNO}_{3}$ with equilibration times of 3 to 4 hours.

\section{A.12 Hayes and Leckje (1987)}

Goethite was synthesized by the method of Atkinson. Posner, and Quirk (1967). The material was characterized by x-ray diffraction. A surface area of $52 \mathrm{~m}^{2} \mathrm{~g}^{-1}$ was determined by the $\mathrm{N}_{2}$ BET method. The surface-site density (7.0 sites $\mathrm{nm}^{2}$ ) was based on a model fit to isotherm data.

\section{A.13 Hsi and Langmuir (1985)}

Goethite and amorphous Fe(III) hydrous oxide were prepared by the methods of Atkinson, Posner, and Quirk (1967) anc Davis and Leckie (1978), respectively. The materials were characterized ty $x$-ray diffraction and scanning electron microscopy. Surface areas of 45 and $306 \mathrm{~m}^{2} \mathrm{~g}^{-1}$ were determined by the $\mathrm{N}_{2}$ BET method for $\alpha$-FeOOH and amorphous iron oxyhydroxide, respectively. The total number of sites $\left(10.5 \times 10^{-3}\right.$ and $1.35 \times 10^{-3}$ mole g.! for $\alpha-F e 00 H$ and amorphous fe(III) hydrous oxide, respectively) were determined by tritium exchange experiments. However, the surface area for amorphous $\mathrm{Fe}($ III) hydrous oxide was treated as ar adjustable mode? parameter. and the value determined was $700 \mathrm{~m}^{2} \mathrm{~g}^{-1}$. These values lead to a site density of 18.1 and 9.0 sites $\mathrm{nm}^{2}$ for $\alpha-F e 00 \mathrm{H}$ and amorphous Fe(III) hydrous oxide. respectively. The value of 20 sites $\mathrm{nm}^{-2}$ for amonhous iron oxyhydroxide reported in Table 2 of Hsi and Langmuir (1985) is based on BET surface area. Adsorption experiments were conducted in $0.1 \mathrm{M} \mathrm{N}^{\mathrm{N}} \mathrm{NO}_{3}$ with equilibration times of 4 hours.

(a) Girvin, D. C..L. L. Ames, A. P. Schwab, and J. E. McGarrah. 1988. "Neptunium Adsorption on Synthetic Amorphous Iron Oxyhydroxide." $J$. colloid Interface sci in press. 


\section{A.14 Leckie et al $(1980)$}

Amorphous Fe(III) hydrous oxide was prepared and characterized as described by Davis and Leckie (1978) except the precipitate was aged for 3 hours at $\mathrm{pH} 7.5$ and for 1 hour at the lowest $\mathrm{pH}$ used in the adsorption experiment. A surface area of $182 \mathrm{~m}^{2} \mathrm{~g}^{-1}$ determined by $\mathrm{N}_{2}$ BET was used to calculate surface charge density. The use of this surface area rather than the $600 \mathrm{~m}^{2} \mathrm{~g}^{-1}$ surface area of Davis and Leckie (1978) requires that the differential capacitance $\left(C_{1}\right.$, Equation 3.1$)$ is much larger than $140 \mu \mathrm{F} \mathrm{cm}^{2}$. Because $p^{*} k^{i n t}$ are for conditions of 0 surface charge, it is not clear what effect (if any) a larger value of $C_{1}$ would have. In addition. Davis and Leckie (1978) point out that the technique for determination of intrinsic surface ionization constants does not require that the specific surface area be known. Adsorption experiments were conducted in $0.1 \mathrm{M} \mathrm{NaNO}_{3}$ with equilibration times of 4 hours. The surface acidity constants apparently are the same as those of Davis and Leckie (1978), although this is not clearly specified.

\section{A.15 Sanchez. Murray, and Sibley (1985)}

Goethite was prepared by the method of Atkinson. Posner, and Ouirk (1967). The surface properties were assumed to be the same as those reported by Balistrieri and Murray (1981). Plutonium redox chemistry is very complicated and controls the design of adsorption experiments. A detailed discussion of the experimental methods are given in Sanche. Murray. and Sibley (1985).

\section{A. 16 Zachara et al $(1987)$}

Amorphous $\mathrm{Fe}$ (III) hydrous oxide was prepared as described by Davis and Leckie (1978) except the precipitate was aged for 14 hours at pH 7.25 . Adsorption experiments were conducted in $0.1 \mathrm{M} \mathrm{NaNO}_{3}$ with equilibration times of 4 hours. Surface parameters used were from Girvin et al. (1988). (a)

(a) Girvin, D. C., L. L. Ames, A. P. Schwab, and J. E. McGarrah. 1988. "Neptunium Adsorption on Synthetic Amorphous Iron Oxyhydroxide." J colloid Interface Sci., in press. 
, 
PNL -6754

DISTRIBUTION

UC-11

No. of

Copies

\section{OFFSITE}

10 DOE/0ffice of Scientific and Technical Information

G. W. Bailey

U.S. Environmental Protection Agency

Environmental Research Laboroatory Athens, GA 30613

D. S. Brown

U.S. Environmental Protection Agency

Environmental Research Laboroatory Athens, GA 30613

A. W. Garrison

U.S. Environmental Protection Agency

Environmental Research Laboroatory Athens, GA 30613

N. T. Loux

U.S. Environmental Protection Agency

Environmenta] Research Laboroatory Athens, GA 30613

L. A. Mulkey

U.S. Environmental Protection Agency

Environmental Research Laboroatory Athens, GA 30613

L. A. Saleem

U.S. Environmenta 1 Protection Agency

Office of Solid Waste

Characterization and Assesment Division

Washington, D.C. 20460
No. of

Copies

C. N. Smith

U.S. Environmental Protection Agency

Environmental Research Laboroatory Athens, GA 30613

R. R. Swank, ur.

U.S. Environmental Protection Agency

Environmenta] Research Laboroatory Athens, GA 30613

QNSITE

DOE Richland Operations office

D. L. Sours

Westinghouse Hanford Company

R. D. Budd

30 Pacific Northwest Laboratory

C. C. Ainsworth

C. E. Cowan

J. W. Falco

J. M. Hales

P. C. Hays

E. A. Jenne

K. M. Krupka

J. P. Mckinley

G. P. O'Connor

R. G. Riley

R. L. Skaggs

R. W. Smith (10)

d. A. Stottlemyre

R. E. Wildung

J. M. Zachara

Publishing Coordination

Technical Report Files (5) 
